Polyhedron

\title{
Tin(IV) compounds of tridentate thiosemicarbazone Schiff bases: synthesis, characterization, in-silico analysis and in vitro cytotoxicity \\ --Manuscript Draft--
}

\begin{tabular}{|c|c|}
\hline Manuscript Number: & \\
\hline Article Type: & Regular Paper \\
\hline Keywords: & Schiff bases, tin(IV); thiosemicarbazone; single-crystal X-ray diffraction; cytotoxicity \\
\hline Corresponding Author: & $\begin{array}{l}\text { Thahira B. S. A. Ravoof } \\
\text { Universiti Putra Malaysia } \\
\text { Selangor, MALAYSIA }\end{array}$ \\
\hline First Author: & Enis Nadia Md Yusof \\
\hline Order of Authors: & Enis Nadia Md Yusof \\
\hline & Alister J. Page \\
\hline & Jennette A. Sakoff \\
\hline & Michela I. Simone \\
\hline & Abhi Veerakumarasivam \\
\hline & Edward R. T. Tiekink \\
\hline & Thahira B. S. A. Ravoof \\
\hline Abstract: & 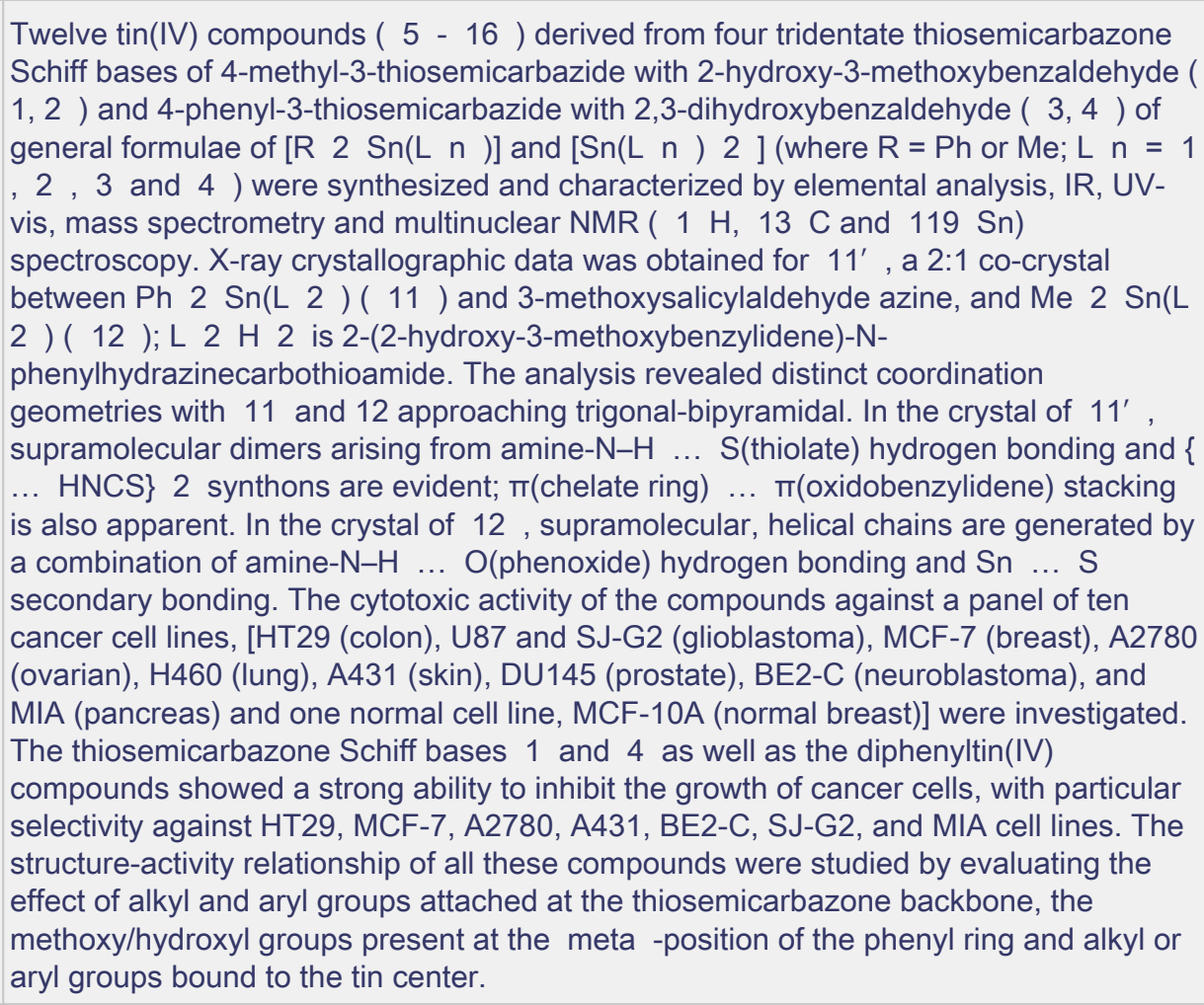 \\
\hline Suggested Reviewers: & $\begin{array}{l}\text { Wan Muhammad Khairul Wan Mohamed Zin } \\
\text { wmkhairul@umt.edu.my } \\
\text { Expert in field }\end{array}$ \\
\hline & $\begin{array}{l}\text { Yang Farina Abdul aziz } \\
\text { farina@ukm.edu.my }\end{array}$ \\
\hline & $\begin{array}{l}\text { Banothile Makhubela } \\
\text { bmakhubela@uj.ac.za }\end{array}$ \\
\hline & Jagadese Vittal \\
\hline
\end{tabular}


chmjjv@nus.edu.sg

Md Uwaisulqarni Osman

uwais@umt.edu.my

Powered by Editorial Manager ${ }^{\circledR}$ and ProduXion Manager ${ }^{\circledR}$ from Aries Systems Corporation 
Editor-in-Chief,

Polyhedron

June $29^{\text {th }}, 2020$

Dear Sir/Madam,

We have just now uploaded corrected files after review, in support of a paper entitled "Tin(IV) compounds of tridentate thiosemicarbazone Schiff bases: synthesis, characterization, in-silico analysis and in vitro cytotoxicity" by Enis Nadia Md Yusof, Alister J. Page, Jennette A. Sakoff, Michela I. Simone, Abhi Veerakumarasivam, Edward R. T. Tiekink and Thahira B. S. A. Ravoof; that we wish to have considered for publication in Polyhedron.

The structural and spectroscopic characterization, as well as the cytotoxicity of the compounds using a panel of cancer cell lines are described and discussed comprehensively.

We hope the submission meets with your Editorial requirements.

Yours sincerely,

Thahira B. S.A. Ravoof

(for the authors) 


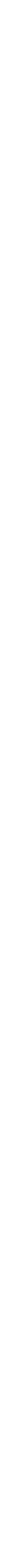

\begin{abstract}
Graphical Abstact - Pictogram (tor revie
\end{abstract}

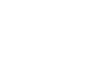
review);Polyhedron_Graphical Abstract.tif

review),Poly actron_Graphical Abstractif
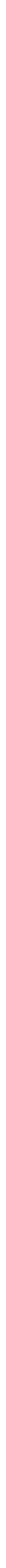


\section{Graphical Abstract}

(1)

\section{Highlights}

- Thiosemicarbazone Schiff bases $\mathbf{1}$ and $\mathbf{4}$ are useful lead candidates for the future organic drug design development to treat cancers.

- Diphenyltin(IV) compounds 5, 8, 11 and $\mathbf{1 4}$ exhibited excellent cytotoxic activity against all cancer cell lines tested. 


\title{
Tin(IV) compounds of tridentate thiosemicarbazone Schiff bases: synthesis, characterization, in-silico analysis and in vitro cytotoxicity
}

Enis Nadia Md Yusof ${ }^{1,2,3}$, Alister J. Page ${ }^{2, *}$, Jennette A. Sakoff ${ }^{4}$, Michela I. Simone ${ }^{2,5}$ Abhi Veerakumarasivam ${ }^{6,7}$, Edward R. T. Tiekink ${ }^{8}$ and Thahira B. S. A. Ravoof ${ }^{1,9, *}$

1 Department of Chemistry, Faculty of Science, Universiti Putra Malaysia, 43400 UPM Serdang, Selangor, Malaysia;

2 Discipline of Chemistry, School of Environmental and Life Sciences, University of Newcastle, University Drive, Callaghan, NSW 2308, Australia;

${ }^{3}$ Chemistry Section, School of Distance Education, Universiti Sains Malaysia, 11800, Minden, Penang, Malaysia

4 Experimental Therapeutics Group, Department of Medical Oncology, Calvary Mater Newcastle Hospital, Edith Street, Waratah NSW 2298, Australia;

5 Priority Research Centre for Drug Development, University of Newcastle, University Drive, Callaghan, NSW 2308, Australia

6 Department of Biological Sciences, School of Science and Technology, Sunway University, No. 5 Jalan Universiti, 47500 Bandar Sunway, Selangor Darul Ehsan, Malaysia;

${ }^{7}$ Medical Genetics Laboratory, Faculty of Medicine and Health Sciences, Universiti Putra Malaysia, 43400 UPM Serdang, Selangor Darul Ehsan, Malaysia

${ }^{8}$ Research Centre for Crystalline Materials, School of Science and Technology, Sunway University, No. 5 Jalan Universiti, 47500 Bandar Sunway, Selangor Darul Ehsan, Malaysia;

${ }^{9}$ Foundry of Reticular Materials for Sustainability (FORMS), Materials Synthesis and Characterization Laboratory, Institute of Advanced Technology, Universiti Putra Malaysia, 43400 Serdang, Selangor Darul Ehsan, Malaysia;

* Correspondence: thahira@upm.edu.my (T.B.S.A.R.), alister.page@newcastle.edu.au (A.J.P.)

\begin{abstract}
Twelve tin(IV) compounds (5-16) derived from four tridentate thiosemicarbazone Schiff bases of 4-methyl-3-thiosemicarbazide with 2-hydroxy-3-methoxybenzaldehyde $(\mathbf{1}, \mathbf{2})$ and 4phenyl-3-thiosemicarbazide with 2,3-dihydroxybenzaldehyde $(\mathbf{3}, 4)$ of general formulae of $\left[\mathrm{R}_{2} \mathrm{Sn}\left(\mathrm{L}^{\mathrm{n}}\right)\right]$ and $\left[\mathrm{Sn}\left(\mathrm{L}^{\mathrm{n}}\right)_{2}\right]$ (where $\mathrm{R}=\mathrm{Ph}$ or $\mathrm{Me} ; \mathrm{L}^{\mathrm{n}}=\mathbf{1}, \mathbf{2}, \mathbf{3}$ and 4) were synthesized and characterized by elemental analysis, IR, UV-vis, mass spectrometry and multinuclear NMR $\left({ }^{1} \mathrm{H},{ }^{13} \mathrm{C}\right.$ and $\left.{ }^{119} \mathrm{Sn}\right)$ spectroscopy. X-ray crystallographic data was obtained for $11^{\prime}$, a $2: 1$ cocrystal between $\mathrm{Ph}_{2} \mathrm{Sn}\left(\mathrm{L}^{2}\right)$ (11) and 3-methoxysalicylaldehyde azine, and $\mathrm{Me}_{2} \mathrm{Sn}\left(\mathrm{L}^{2}\right)(\mathbf{1 2})$;
\end{abstract}


$\mathrm{L}^{2} \mathrm{H}_{2}$ is 2-(2-hydroxy-3-methoxybenzylidene)-N-phenylhydrazinecarbothioamide. The analysis revealed distinct coordination geometries with $\mathbf{1 1}$ and 12 approaching trigonalbipyramidal. In the crystal of 11', supramolecular dimers arising from amine-N$\mathrm{H}^{\cdots} \mathrm{S}$ (thiolate) hydrogen bonding and $\{\cdots \mathrm{HNCS}\}_{2}$ synthons are evident; $\pi$ (chelate ring) ${ }^{\cdots} \pi$ (oxidobenzylidene) stacking is also apparent. In the crystal of $\mathbf{1 2}$, supramolecular, helical chains are generated by a combination of amine- $\mathrm{N}-\mathrm{H} \cdots \mathrm{O}$ (phenoxide) hydrogen bonding and $\mathrm{Sn}^{\cdots} \mathrm{S}$ secondary bonding. The cytotoxic activity of the compounds against a panel of ten cancer cell lines, [HT29 (colon), U87 and SJ-G2 (glioblastoma), MCF-7 (breast), A2780 (ovarian), H460 (lung), A431 (skin), DU145 (prostate), BE2-C (neuroblastoma), and MIA (pancreas) and one normal cell line, MCF-10A (normal breast)] were investigated. The thiosemicarbazone Schiff bases $\mathbf{1}$ and $\mathbf{4}$ as well as the diphenyltin(IV) compounds showed a strong ability to inhibit the growth of cancer cells, with particular selectivity against HT29, MCF-7, A2780, A431, BE2-C, SJ-G2, and MIA cell lines. The structure-activity relationship of all these compounds were studied by evaluating the effect of alkyl and aryl groups attached at the thiosemicarbazone backbone, the methoxy/hydroxyl groups present at the meta-position of the phenyl ring and alkyl or aryl groups bound to the tin center.

\section{Introduction}

Schiff bases that contain nitrogen, sulfur and oxygen as donor atoms such as thiosemicarbazones, semicarbazones, and dithiocarbazates and their metal complexes have been of interest since 1946 [1], owing to their remarkable biological and pharmacological properties, especially antitumor, antibacterial, antiviral, anti-tuberculosis, antifungal, and antimalarial activities [2], that are altered when small changes to the structures (e.g., changing of functional group) are applied. Thiosemicarbazones are considered as privileged ligands due to their potential donor atoms, $\pi$-delocalization and configurational flexibility that can produce various metal-ligand linkages [3]. Compounds having thiol groups have also been proven to inhibit the ribonucleotide reductase (RR) enzyme, used in DNA synthesis. Hence, by inhibiting or blocking the function of the RR enzyme, the DNA replication and synthesis of tumor cells can be controlled or prevented [4]. In many cases, complexation with metal ions increased the bioactivity of the compounds suggesting that coordination of such ligands enhanced cytotoxicity. 
Although much research has been devoted to the synthesis, characterization and biological properties of ligands coordinated to transition metal ions. Tin-based compounds have received considerably less attention because of ecotoxicology effects at the biochemical, cellular, and organism level [5]. In recent years, tin-based compounds have been of great interest because of their ability to form stable bonds with hetero donor atoms, for instance nitrogen, sulfur and oxygen atoms [6-15]. Tin(IV) compounds are now well-known for their applications as cytotoxic, biocidal, antibacterial and antifungal agents [6,16-19]. Many studies have reported the antimicrobial activities of tin(IV) compounds derived from thiosemicarbazone Schiff bases. In particular, compounds containing the 3methoxysalicylaldehyde thiosemicarbazone Schiff base were tested for their in vitro cytotoxicity against human acute lymphoblastic leukemia (Jurkat cells) [20]. The data indicated increasing potency, in the order of dimethyltin(IV) $<$ diphenyltin(IV) < dibutyltin(IV) compounds, with $\mathrm{IC}_{50}$ values of 260,130 , and $50 \mu \mathrm{M}$, respectively. This suggested that the cytotoxicity of dialkytin(IV) compounds increased with the increase in the length of the organic chain. The cytotoxicity of diphenyl- and dimethyltin(IV) compounds of pyruvic acid thiosemicarbazone have also been investigated against human breast adenocarcinoma (MCF-7), bladder carcinoma (T24), non-small cell lung carcinoma (A-549) and mouse fibroblast (L-929) cell lines with $\mathrm{IC}_{50}$ values in the range of 0.43 to $19.73 \mu \mathrm{M}$. The diphenyltin(IV) compound was most potent against T-24 cells with an $\mathrm{IC}_{50}$ value of $0.43 \mu \mathrm{M}$, where it exhibited 96-fold better activity than cisplatin [21].

Recently, tin(IV) compounds derived from tridentate 2-hydroxy-5-methoxybenzaldehyde$\mathrm{N}(4)$-methylthiosemicarbazone exhibited higher anticancer activity against the human colorectal (HTC-116) cell line as compared to the reference drug, 5-fluorouracil [8]. The significant biological activity of the tin(IV) compounds were influenced by the types of organo group attached to the tin center, diffusion, lipophilicity, and steric effects $[8,22-24]$.

As a continuation of our research on tridentate ONS Schiff bases and their tin(IV) compounds $[25,26]$, we report herein the preparation, spectroscopic characterization, and bioactivity of tin(IV) compounds (5-16) containing 2-hydroxy-3-methoxybenzyl- and 2,3-dihydroxybenzylderived thiosemicarbazone Schiff bases (1-4). Diphenyl- (5, 8, 11, and 14) and dimethyltin(IV) $(\mathbf{6}, \mathbf{9}, \mathbf{1 2}$, and $\mathbf{1 5})$ compounds exhibited penta-coordinated geometry whereas tin(IV) compounds were coordinated to two molecules of thiosemicarbazone Schiff bases (7, 10, 13, and 16) suggesting a hexa-coordinated geometry according to ${ }^{119} \mathrm{Sn}$ NMR analysis. 
The experimental data (FTIR, electronic and single crystal X-ray diffraction analysis) of the

\section{Experimental}

\subsection{Physical Measurements}

Melting points were determined using an Electrothermal digital melting point apparatus. IR spectra were recorded using the Perkin Elmer Spectrum 100 with Universal ATR Polarization in the range 4000-280 $\mathrm{cm}^{-1}$. C, $\mathrm{H}$ and $\mathrm{N}$ elemental analyses were carried out using a LECO CHNS-932 instrument. Molar conductivities of $10^{-3} \mathrm{M}$ solutions of the organotin(IV) compounds in DMSO were measured at $27{ }^{\circ} \mathrm{C}$ using a Jenway 4310 conductivity meter fitted with a dip-type cell with a platinized electrode. Electronic spectra were recorded on a Shimadzu UV-1650 PC recording spectrophotometer (1000-200 nm). ${ }^{1} \mathrm{H}-$ and ${ }^{13} \mathrm{C}-\mathrm{NMR}$ spectra were recorded using an NMR JNM ECA400 spectrometer. ${ }^{119} \mathrm{Sn}$ NMR were measured using a Bruker BioSpin Avance III (600MHz) spectrometer. The mass spectra were recorded using a Shimadzu GC-MS QP2010Plus mass spectrometer.

\subsection{Materials}


All solvents and reagents were of analytical reagent grade and used without further purification.

Chemicals: 4-methyl-3-thiosemicarbazide, 4-phenyl-3-thiosemicarbazide, potassium hydroxide, 2-hydroxy-3-methoxybenzaldehyde, 2,3-dihydroxybenzaldehyde, dichlorodiphenyltin(IV), dichlorodimethyltin(IV), tin(II) chloride. Solvents: absolute ethanol, 99.8\%, ethanol, $95 \%$, methanol and dimethylsulfoxide.

\subsection{Syntheses}

2.3.1. Syntheses of 2-(2-hydroxy-3-methoxybenzylidene)-N-methylhydrazinecarbothioamide (1) and 2-(2-hydroxy-3-methoxybenzylidene)-N-phenylhydrazinecarbothioamide (2)

Compounds 1 and $\mathbf{2}$ were prepared according to the procedure described in the literature $[27,28]$ with some modifications.

4-Methylthiosemicarbazide (1.05 g, $10 \mathrm{mmol}) / 4$-phenylthiosemicarbazide (1.67 g, $10 \mathrm{mmol})$ was dissolved in methanol $\left(40 \mathrm{~cm}^{3}\right)$ with stirring and heating $\left(40^{\circ} \mathrm{C}\right)$ over a period of 30 minutes. 3-Methoxysalicylaldehyde $(1.52 \mathrm{~g}, 10 \mathrm{mmol})$ in $10 \mathrm{~cm}^{3}$ of methanol was added to the thiosemicarbazide solution and stirred at room temperature for $4 \mathrm{~h}$. Upon cooling, a crystalline product began to form which was filtered, washed with cold methanol, and dried in a desiccator over anhydrous silica gel.

\section{2-(2-hydroxy-3-methoxybenzylidene)-N-methylhydrazinecarbothioamide (1)}

Colorless crystalline solid. Yield: $92 \%$. Melting point: $242-243^{\circ} \mathrm{C}$. Analysis calculated for $\mathrm{C}_{10} \mathrm{H}_{13} \mathrm{~N}_{3} \mathrm{O}_{2} \mathrm{~S}$ : C, 50.19; H, 5.48; N, 17.56. Found: C, 49.93; H, 5.38; N, $17.22 \%$. FT-IR $\left(\mathrm{ATR}, \mathrm{cm}^{-1}\right): 3337 v(\mathrm{OH}), 3304 v(\mathrm{NH}), 1610 v(\mathrm{C}=\mathrm{N}), 1109 v(\mathrm{~N}-\mathrm{N}), 1037 v(\mathrm{C}=\mathrm{S}) .{ }^{1} \mathrm{H}-\mathrm{NMR}$ (DMSO-d 6 ) $\delta$ (ppm.): 2.99 (d, 3H, $\mathrm{CH}_{3}$ ), 3.79 (s, 3H, O-CH $\mathrm{CH}_{3}, 6.93-7.54$ (m, 3H, Ar-H), 8.37 (s, 1H, CH), 8.39 (q, 1H, C(=S)-NH), 9.18 (s, 1H, OH), 11.42 (s, 1H, NH-N). ${ }^{13} \mathrm{C}$ NMR (DMSO-d $) \delta$ (ppm.): $30.8\left(\mathrm{~N}-\mathrm{CH}_{3}\right), 56.4\left(\mathrm{O}_{-} \mathrm{CH}_{3}\right), 113.1,118.2,119.2,121.2,139.6,146.7$ (Ar-C), $148.4(\mathrm{C}=\mathrm{N}), 177.6(\mathrm{C}=\mathrm{S})$.

\section{2-(2-hydroxy-3-methoxybenzylidene)-N-phenylhydrazinecarbothioamide (2)}

White crystalline solid. Yield: $90 \%$. Melting point: $209-210^{\circ} \mathrm{C}$. Analysis calculated for $\mathrm{C}_{15} \mathrm{H}_{15} \mathrm{~N}_{3} \mathrm{O}_{2} \mathrm{~S}$ : C, 59.78; H, 5.02; N, 13.94. Found: C, 59.99; H, 5.15; N, $13.80 \%$ \% FT-IR $\left(\right.$ ATR, $\left.\mathrm{cm}^{-1}\right): 3300 v(\mathrm{NH}), 1609 v(\mathrm{C}=\mathrm{N}), 1103 v(\mathrm{~N}-\mathrm{N}), 908 v(\mathrm{C}=\mathrm{S}) .{ }^{1} \mathrm{H}$ NMR (DMSO-d 6$) \delta$

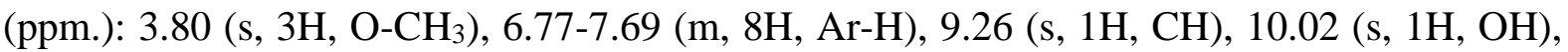


$11.78(\mathrm{~s}, 1 \mathrm{H}, \mathrm{NH}-\mathrm{N}) .{ }^{13} \mathrm{C}$ NMR (DMSO-d 6$) \delta$ (ppm.): $56.3\left(\mathrm{O}-\mathrm{CH}_{3}\right), 113.4,118.8,119.5$, 121.2, 125.4, 126.4, 128.6, 139.7, 140.4, $146.6(10 \mathrm{x}$ aromatic-C $), 148.6(\mathrm{C}=\mathrm{N}), 176.1(\mathrm{C}=\mathrm{S})$.

\subsubsection{Syntheses of 2-(2,3-dihydroxybenzylidene)-N-methylhydrazinecarbothioamide (3) and} 2-(2,3-dihydroxybenzylidene)-N-phenylhydrazinecarbothioamide (4)

Compounds 3 and $\mathbf{4}$ were prepared according to the procedure described in the literature [29,30]. A $25 \mathrm{~cm}^{3}$ ethanolic solution of 2,3-dihydroxybenzaldehyde (1.38 g, $\left.10 \mathrm{mmol}\right)$ was added to an equimolar ethanolic solution $\left(10 \mathrm{~cm}^{3}\right)$ of 4-methyl-3-thiosemicarbazide $(1.05 \mathrm{~g}$, $10 \mathrm{mmol}) / 4$-phenyl-3-thiosemicarbazide $(1.67 \mathrm{~g}, 10 \mathrm{mmol})$. The mixture was stirred for 3 hours at room temperature and the title compound was filtered. The title compound was then recrystallized from methanol to remove all the impurities and kept in desiccator over anhydrous silica gel.

\section{2-(2,3-dihydroxybenzylidene)-N-methylhydrazinecarbothioamide (3)}

Pale yellow solid. Yield: $83 \%$. Melting point: $231-232^{\circ} \mathrm{C}$. Analysis calculated for $\mathrm{C}_{9} \mathrm{H}_{11} \mathrm{~N}_{3} \mathrm{O}_{2} \mathrm{~S}$ : C, 47.99; H, 4.92; N, 18.65. Found: C, 46.88; H, 4.85; N, $18.38 \%$. FT-IR $\left(\mathrm{ATR}, \mathrm{cm}^{-1}\right): 3418 v(\mathrm{OH}), 3140 v(\mathrm{NH}), 1601 v(\mathrm{C}=\mathrm{N}), 1112 v(\mathrm{~N}-\mathrm{N}), 1035 v(\mathrm{C}=\mathrm{S}) .{ }^{1} \mathrm{H}$ NMR (DMSO-d 6 ) $\delta$ (ppm.): 3.00 (d, 3H, $\left.\mathrm{CH}_{3}\right), 6.67-7.38$ (m, 3H, Ar-H), 9.02 (s, 1H, CH), 8.37, $8.38(2 \times$ s $, 2 \mathrm{H}, \mathrm{OH}), 9.49$ (s, 1H, C(=S)-NH), 11.40 (s, 1H, NH-N). ${ }^{13} \mathrm{C}$ NMR (DMSO-d 6 ) $\delta$ (ppm.): $31.3\left(\mathrm{~N}_{-} \mathrm{CH}_{3}\right), 116.7,117.4,119.4,121.5,140.1,145.6(\mathrm{Ar}-\mathrm{C}), 146.0(\mathrm{C}=\mathrm{N}), 178.0$ $(\mathrm{C}=\mathrm{S})$.

\section{2-(2,3-dihydroxybenzylidene)-N-phenylhydrazinecarbothioamide (4)}

Pale yellow solid. Yield: $70 \%$. Melting point: $215-216^{\circ} \mathrm{C}$. Analysis calculated for $\mathrm{C}_{14} \mathrm{H}_{13} \mathrm{~N}_{3} \mathrm{O}_{2} \mathrm{~S}$ : C, 58.52; H, 4.56; N, 14.62. Found: C, 57.61; H, 4.69; N, $14.67 \%$. FT-IR $\left(\right.$ ATR, $\left.\mathrm{cm}^{-1}\right): 3443 v(\mathrm{OH}), 3129 v(\mathrm{NH}), 1597 v(\mathrm{C}=\mathrm{N}), 1047 v(\mathrm{~N}-\mathrm{N}), 1029 v(\mathrm{C}=\mathrm{S}) .{ }^{1} \mathrm{H}$ NMR (DMSO-d $)_{6} \delta$ (ppm.): 6.67-7.57 (m, 8H, Ar-H), 8.49 (s, 1H, CH), 8.96, 9.52 (2 x s, 2H, OH), 10.01 (s, $1 \mathrm{H}, \mathrm{C}(=\mathrm{S})-\mathrm{NH}), 11.75$ (s, $1 \mathrm{H}, \mathrm{NH}-\mathrm{N}) .{ }^{13} \mathrm{C}$ NMR (DMSO-d 6 ) $\delta$ (ppm.): 117.1, 117.9, $119.5,121.3,125.6,126.0,128.5,139.6,141.3,145.9(A r-C), 146.0(\mathrm{C}=\mathrm{N}), 176.0(\mathrm{C}=\mathrm{S})$. 


\subsubsection{General procedure for the synthesis of tin(IV) compounds derived from 1 and $\mathbf{3}$}

To a solution of $1(0.24 \mathrm{~g}, 1 \mathrm{mmol}) / 3(0.23 \mathrm{~g}, 1 \mathrm{mmol})$ in $100 \mathrm{~cm}^{3}$ of methanol, $\mathrm{KOH}(0.11$ $\mathrm{g}, 2 \mathrm{mmol}$ ) was added and the mixture was stirred and heated for 30 minutes in methanol. Then, $1 \mathrm{mmol}$ of tin precursor $\left(\mathrm{Ph}_{2} \mathrm{SnCl}_{2}(0.34 \mathrm{~g}) / \mathrm{Me}_{2} \mathrm{SnCl}_{2}(0.22 \mathrm{~g}) / \mathrm{SnCl}_{2}(0.19 \mathrm{~g})\right)$ was added to the mixture and refluxed for 2 hours under nitrogen. The mixture was filtered while hot and then the filtrate was placed in the freezer until a bright yellow solid formed. The solid residue obtained was recrystallized from methanol.

Diphenyltin(IV) compound of 2-(2-hydroxy-3-methoxybenzylidene)-N-methylhydrazine carbothioamide (5)

Bright yellow solid. Yield: $74 \%$. Melting point: $138-139^{\circ} \mathrm{C}$. Analysis calculated for $\mathrm{C}_{22} \mathrm{H}_{21} \mathrm{~N}_{3} \mathrm{O}_{2} \mathrm{SSn}$ : C, 51.79; H, 4.15; N, 8.24. Found: C, 51.03; H, 4.23; N, $8.16 \%$. FT-IR $\left(\mathrm{ATR}, \mathrm{cm}^{-1}\right): 3299 v(\mathrm{~N}-\mathrm{H}), 1596 v(\mathrm{C}=\mathrm{N}), 1066 v(\mathrm{~N}-\mathrm{N}), 973 v(\mathrm{C}=\mathrm{S}) .{ }^{1} \mathrm{H}$ NMR $\left(\mathrm{CDCl}_{3}\right) \delta$ (ppm.): 3.01 (d, 3H, N-CH $), 3.96$ (s, 3H, O-CH 3 ), 7.96 (s, 1H, CH), 7.36-8.12 (m, 13H, Ar$\mathrm{H}), 8.59(\mathrm{~s}, 1 \mathrm{H}, \mathrm{NH}) .{ }^{13} \mathrm{C} \mathrm{NMR}\left(\mathrm{CDCl}_{3}\right) \delta\left(\mathrm{ppm}\right.$.): $29.8\left(\mathrm{NH}-\mathrm{CH}_{3}\right), 56.5\left(\mathrm{O}-\mathrm{CH}_{3}\right), 115.7$, 116.7, 117.2, 125.1, 128.6, 129.9, 135.9, 142.5, 151.6 (Ar-C), $157.1(\mathrm{C}=\mathrm{N}), 160.3$ (S-C-S). ${ }^{119} \mathrm{Sn} \operatorname{NMR}\left(\mathrm{CDCl}_{3}\right) \delta$ (ppm.): -236.

Dimethyltin(IV) compound of 2-(2-hydroxy-3-methoxybenzylidene)-N-methylhydrazine carbothioamide (6)

Bright yellow solid. Yield: $42 \%$. Melting point: $164-168^{\circ} \mathrm{C}$. Analysis calculated for $\mathrm{C}_{12} \mathrm{H}_{17} \mathrm{~N}_{3} \mathrm{O}_{2} \mathrm{SSn}$ : C, 37.33; H, 4.44; N, 10.88. Found: C, 39.00; H, 4.76; N, $11.00 \%$. FT-IR (ATR, $\left.\mathrm{cm}^{-1}\right): 3222 v(\mathrm{~N}-\mathrm{H}), 1590 v(\mathrm{C}=\mathrm{N}), 1066 v(\mathrm{~N}-\mathrm{N}), 973 v(\mathrm{C}=\mathrm{S}) .{ }^{1} \mathrm{H} \mathrm{NMR}\left(\mathrm{CDCl}_{3}\right) \delta$ (ppm.): 0.91 (s, 6H, Sn-CH CH $_{3}, 2.97$ (d, 3H, N-CH $), 3.94$ (s, 3H, O-CH $\mathrm{CH}_{3}, 7.26$ (s, 1H, CH), 6.66-6.87 (m, 3H, Ar-H), 8.55 (s, 1H, NH). ${ }^{13} \mathrm{C}$ NMR $\left(\mathrm{CDCl}_{3}\right) \delta$ (ppm.): $8.7\left(\mathrm{Sn}_{-} \mathrm{CH}_{3}\right), 31.3$ $\left(\mathrm{NH}-\mathrm{CH}_{3}\right), 56.4\left(\mathrm{O}-\mathrm{CH}_{3}\right), 115.7,115.8,117.8,118.5,125.8,151.3(\mathrm{Ar}-\mathrm{C}), 156.5(\mathrm{C}=\mathrm{N})$, 178.0 (S-C-S). ${ }^{119}$ Sn NMR (DMSO-d 6 ) $\delta$ (ppm.): -154.

Tin(IV) compound of 2-(2-hydroxy-3-methoxybenzylidene)-N-methylhydrazine carbothioamide (7)

Compound $\mathbf{7}$ was prepared following the same procedure as described for $\mathbf{5}$, using $\mathbf{1}(0.48 \mathrm{~g}$, 2 mmol). Bright yellow solid. Yield: $31 \%$. Melting point: $118-119^{\circ} \mathrm{C}$. Analysis calculated for $\mathrm{C}_{20} \mathrm{H}_{22} \mathrm{~N}_{6} \mathrm{O}_{4} \mathrm{~S}_{2} \mathrm{Sn}: \mathrm{C}, 40.49 ; \mathrm{H}, 3.74 ; \mathrm{N}, 14.17$. Found: C, 40.50; H, 3.33; N, $14.17 \%$. FT-IR 
$\left(\right.$ ATR, $\left.\mathrm{cm}^{-1}\right): 3308 v(\mathrm{~N}-\mathrm{H}), 1590 v(\mathrm{C}=\mathrm{N}), 1066 v(\mathrm{~N}-\mathrm{N}), 973 v(\mathrm{C}=\mathrm{S}) .{ }^{1} \mathrm{H}$ NMR $\left(\mathrm{DMSO}^{-} \mathrm{d}_{6}\right) \delta$ (ppm.): 2.36 (d, 6H, N-CH3), $3.82\left(\mathrm{~s}, 6 \mathrm{H}, \mathrm{O}-\mathrm{CH}_{3}\right), 7.60$ (s, 2H, CH), 6.67-7.31 (m, 6H, Ar$\mathrm{H}), 8.88$ (s, 2H, NH). ${ }^{13} \mathrm{C}$ NMR (DMSO-d 6$) \delta\left(\mathrm{ppm}\right.$ ) : $19.3\left(\mathrm{NH}-\mathrm{CH}_{3}\right), 56.7\left(\mathrm{O}_{-} \mathrm{CH}_{3}\right), 105.4$, 116.7, 117.4, 126.5, 128.6, 129.0, 130.7, 130.8, 134.9, 149.7, 151.8, 158.3 (Ar-C), 163.7 $(\mathrm{C}=\mathrm{N}), 170.6(\mathrm{~S}-\mathrm{C}-\mathrm{S}) .{ }^{119} \mathrm{Sn}$ NMR (DMSO-d $) \delta$ (ppm.): -354 .

Diphenyltin(IV) compound of 2-(2,3-dihydroxybenzylidene)-N-methylhydrazine carbothioamide (8)

Yellow solid. Yield: $49 \%$. Melting point: $186-192^{\circ} \mathrm{C}$. Analysis calculated for $\mathrm{C}_{21} \mathrm{H}_{19} \mathrm{~N}_{3} \mathrm{O}_{2} \mathrm{SSn}$ : C, 50.83; H, 3.86; N, 8.47. Found: C, 48.06; H, 4.27; N, $8.02 \%$. FT-IR $\left(\right.$ ATR, $\left.\mathrm{cm}^{-1}\right): 1593 v(\mathrm{C}=\mathrm{N}), 1006 v(\mathrm{~N}-\mathrm{N}), 953 v(\mathrm{C}=\mathrm{S}) .{ }^{1} \mathrm{H}$ NMR $\left(\mathrm{DMSO}-\mathrm{d}_{6}\right) \delta(\mathrm{ppm}$ ) $): 00$ (s, 3H, NH-CH 3$), 8.57$ (s, 1H, CH), 6.37-8.20 (m, 13H, Ar-H), 11.27 (s, 1H, NH). ${ }^{13} \mathrm{C}$ NMR $\left(\mathrm{DMSO}-\mathrm{d}_{6}\right) \delta$ (ppm.): $30.9\left(\mathrm{NH}-\mathrm{CH}_{3}\right), 112.8,113.7,115.7,118.4,128.3,129.2,136.0,136.1$, 141.1, 145.4, 153.3 (aromatic-C), $154.3(\mathrm{C}=\mathrm{N}), 177.0(\mathrm{~S}-\mathrm{C}-\mathrm{S}) .{ }^{119} \mathrm{Sn}$ NMR (DMSO-d 6 ) $\delta$ (ppm.): -227.

Dimethyltin(IV) compound of 2-(2,3-dihydroxybenzylidene)-N-methylhydrazine carbothioamide (9)

Yellow solid. Yield: $32 \%$. Melting point: $223-225^{\circ} \mathrm{C}$. Analysis calculated for $\mathrm{C}_{11} \mathrm{H}_{15} \mathrm{~N}_{3} \mathrm{O}_{2} \mathrm{SSn}$ : C, 35.51; H, 4.06; N, 11.29. Found: C, 35.87; H, 3.86; N, $11.35 \%$. FT-IR $\left(\right.$ ATR, $\left.\mathrm{cm}^{-1}\right): 1596 v(\mathrm{C}=\mathrm{N}), 1006 v(\mathrm{~N}-\mathrm{N}), 951 v(\mathrm{C}=\mathrm{S}) .{ }^{1} \mathrm{H}$ NMR (DMSO-d 6 ) $\delta$ (ppm.): 0.62 (s, 6H, Sn-CH $\mathrm{CH}_{3}, 3.00$ (d, 3H, N-CH $), 6.32-7.10$ (m, 3H, Ar-H), 8.38 (s, 1H, CH), 11.30 (s, $1 \mathrm{H}, \mathrm{NH}) .{ }^{13} \mathrm{C}$ NMR (DMSO-d 6$) \delta$ (ppm.): $6.8\left(\mathrm{Sn}_{\mathrm{C}} \mathrm{CH}_{3}\right), 30.7\left(\mathrm{NH}-\mathrm{CH}_{3}\right), 112.6,113.7,115.6$, 118.6, 140.7, 153.9 (Ar-C), $155.0(\mathrm{C}=\mathrm{N}), 177.2$ (S-C-S). ${ }^{119}$ Sn NMR (DMSO-d 6$) \delta$ (ppm.): 123.

Tin(IV) compound of 2-(2,3-dihydroxybenzylidene)-N-methylhydrazinecarbothioamide (10)

Compound $\mathbf{1 0}$ was prepared following the same procedure as described for $\mathbf{5}$, using $\mathbf{1}$ ( $0.46 \mathrm{~g}$, $2 \mathrm{mmol}$ ). Orange solid. Yield: $79 \%$. Melting point: $>300^{\circ} \mathrm{C}$. Analysis calculated for $\mathrm{C}_{18} \mathrm{H}_{18} \mathrm{~N}_{6} \mathrm{O}_{4} \mathrm{~S}_{2} \mathrm{Sn}$ : C, 38.25; H, 3.21; N, 14.87. Found: C, 36.62; H, 2.92; N, $14.21 \%$. FT-IR $\left(\mathrm{ATR}, \mathrm{cm}^{-1}\right): 1585 v(\mathrm{C}=\mathrm{N}), 993 v(\mathrm{~N}-\mathrm{N}), 951 v(\mathrm{C}=\mathrm{S}) .{ }^{1} \mathrm{H}$ NMR (DMSO-d 6$) \delta(\mathrm{ppm}$.): $2.83(\mathrm{~s}$, $\left.3 \mathrm{H}, \mathrm{N}-\mathrm{CH}_{3}\right), 6.65-7.52$ (m, 6H, Ar-H), 8.34 (s, 1H, OH), 8.77 (s, 1H, CH), 11.27 (s, 1H, NH). 
${ }^{13} \mathrm{C}$ NMR (DMSO-d $) \delta$ (ppm.): $30.6\left(\mathrm{NH}-\mathrm{CH}_{3}\right), 113.8,114.3,116.7,118.9,140.0 .150 .1$ (Ar-C), $150.6(\mathrm{C}=\mathrm{N}), 177.5(\mathrm{~S}-\mathrm{C}-\mathrm{S}) .{ }^{119} \mathrm{Sn}$ NMR $\left(\mathrm{DMSO}^{\left.-\mathrm{d}_{6}\right)} \delta(\mathrm{ppm}): 519\right.$.

\subsubsection{General procedure for the syntheses of tin(IV) compounds derived from 2}

Compound $2(0.30 \mathrm{~g}, 1 \mathrm{mmol})$ was dissolved in methanol $\left(100 \mathrm{~cm}^{3}\right)$ and $\mathrm{Et}_{3} \mathrm{~N}\left(0.28 \mathrm{~cm}^{3}, 2\right.$ mmol) was added dropwise to the solution of 2 . The mixture was heated $\left(40^{\circ} \mathrm{C}\right)$ for about 2 hours until the solution was reduced by half. Next $1 \mathrm{mmol}$ of tin precursor $\left(\mathrm{Ph}_{2} \mathrm{SnCl}_{2}(0.34\right.$ $\left.\mathrm{g}) / \mathrm{Me}_{2} \mathrm{SnCl}_{2}(0.22 \mathrm{~g}) / \mathrm{SnCl}_{2}(0.19 \mathrm{~g})\right)$ was added to the mixture. The mixture was refluxed under nitrogen for about 2 hours and filtered while hot to remove triethylamine salt and the filtrate was kept at room temperature until a bright yellow product formed.

Diphenyltin(IV) compound of 2-(2-hydroxy-3-methoxybenzylidene)-N-phenylhydrazine carbothioamide (11)

Yellow crystals. Yield: $73 \%$. Melting point: $205-207^{\circ} \mathrm{C}$. Analysis calculated for $\mathrm{C}_{27} \mathrm{H}_{23} \mathrm{~N}_{3} \mathrm{O}_{2} \mathrm{SSn}$ : C, 56.67; H, 4.05; N, 7.34\%. Found: C, 57.53; H, 4.26; N, 7.87\%. FT-IR $\left(\mathrm{ATR}, \mathrm{cm}^{-1}\right): 3331 v(\mathrm{~N}-\mathrm{H}), 1586 v(\mathrm{C}=\mathrm{N}), 1075 v(\mathrm{~N}-\mathrm{N}), 832 v(\mathrm{C}=\mathrm{S}) .{ }^{1} \mathrm{H}$ NMR $\left(\mathrm{CDCl}_{3}\right) \delta$ (ppm.): 3.96 (s, 3H, O-CH ${ }_{3}$ ), 7.99 (s, 1H, CH), 6.69-7.56 (m, 18H, Ar-H), 8.70 (s, 1H, NH). ${ }^{13} \mathrm{C} \mathrm{NMR}\left(\mathrm{CDCl}_{3}\right) \delta$ (ppm.): $56.7\left(\mathrm{O}-\mathrm{CH}_{3}\right), 115.2,116.2,116.9,119.4,120.7,123.4,124.1$, $125.4,128.7,128.9,130.0,135.9,139.3,142.1,148.3,149.7,151.7($ Ar-C $), 162.5(\mathrm{C}=\mathrm{N})$, 164.8 (S-C-S). ${ }^{119} \mathrm{Sn}$ NMR $\left(\mathrm{CDCl}_{3}\right) \delta$ (ppm.): -242.

Dimethyltin(IV) compound of 2-(2-hydroxy-3-methoxybenzylidene)-N-phenylhydrazine carbothioamide (12)

Yellow crystals. Yield: $64 \%$. Melting point: $176-179^{\circ} \mathrm{C}$. Analysis calculated for $\mathrm{C}_{17} \mathrm{H}_{19} \mathrm{~N}_{3} \mathrm{O}_{2} \mathrm{SSn}: \mathrm{C}, 45.56 ; \mathrm{H}, 4.27 ; \mathrm{N}, 9.38 \%$. Found: C, 45.86; H, 4.40; N, 7.08\%. FT-IR $\left(\mathrm{ATR}, \mathrm{cm}^{-1}\right): 3294 v(\mathrm{~N}-\mathrm{H}), 1577 v(\mathrm{C}=\mathrm{N}), 1059 v(\mathrm{~N}-\mathrm{N}), 824 v(\mathrm{C}=\mathrm{S}) .{ }^{1} \mathrm{H}$ NMR $\left(\mathrm{CDCl}_{3}\right) \delta$ (ppm.): 3.85 (s, 3H, O-CH3), 7.53 (s, 1H, CH), 6.69-7.32 (m, 8H, Ar-H), 8.65 (s, 1H, NH). ${ }^{13} \mathrm{C} \mathrm{NMR}\left(\mathrm{CDCl}_{3}\right) \delta$ (ppm.): $6.5\left(\mathrm{Sn}-\mathrm{CH}_{3}\right), 56.2\left(\mathrm{O}_{-} \mathrm{CH}_{3}\right), 115.3,116.6,116.7,120.5,123.3$, 125.4, 128.9, 139.4, 151.3, $156.8(\mathrm{Ar}-\mathrm{C}), 162.5(\mathrm{C}=\mathrm{N}), 163.9(\mathrm{~S}-\mathrm{C}-\mathrm{S}) .{ }^{119} \mathrm{Sn} \mathrm{NMR}\left(\mathrm{CDCl}_{3}\right) \delta$ (ppm.): -115 .

Tin(IV) compound of 2-(2-hydroxy-3-methoxybenzylidene)-N-phenylhydrazine carbothioamide (13) 
Compound 13 was prepared following the same procedure as described for 11, using 2 (0.60 g, 2 mmol). Yellow solid. Yield: $50 \%$. Melting point: $293-294^{\circ} \mathrm{C}$. Analysis calculated for $\mathrm{C}_{30} \mathrm{H}_{26} \mathrm{~N}_{6} \mathrm{O}_{4} \mathrm{~S}_{2} \mathrm{Sn}: \mathrm{C}, 50.23 ; \mathrm{H}, 3.65 ; \mathrm{N}, 11.71 \%$. Found: C, 49.85; H, 3.73; N, $11.60 \%$. FT-IR $\left(\mathrm{ATR}, \mathrm{cm}^{-1}\right): 3303 v(\mathrm{~N}-\mathrm{H}), 1581 v(\mathrm{C}=\mathrm{N}), 1063 v(\mathrm{~N}-\mathrm{N}), 824 v(\mathrm{C}=\mathrm{S}) .{ }^{1} \mathrm{H}$ NMR (DMSO-d 6$) \delta$ (ppm.): 3.58 (s, 6H, O-CH $), 9.08$ (s, 2H, CH), 6.80-7.73 (m, 16H, Ar-H), 9.70 (s, 2H, NH). ${ }^{13} \mathrm{C}$ NMR (DMSO-d $\left.\mathrm{d}_{6}\right) \delta$ (ppm.): $56.4\left(\mathrm{O}-\mathrm{CH}_{3}\right), 117.9,118.2,121.0,123.4,126.8,129.2$, 140.4, 151.4, 154.8 (Ar-C), $160.3(\mathrm{C}=\mathrm{N}), 162.2$ (S-C-S). ${ }^{119}$ Sn NMR (DMSO-d 6 ) $\delta$ (ppm.): 451.

\subsubsection{General procedure for the synthesis of tin(IV) compounds derived from 4}

Compound $4(0.29 \mathrm{~g}, 0.001 \mathrm{~mol})$ was dissolved in methanol $\left(100 \mathrm{~cm}^{3}\right)$ and $\mathrm{KOH}(0.11 \mathrm{~g}, 2$ mmol) was added dropwise to the solution of 4 . The mixture was refluxed for about 30 minutes, where the color changed from light yellow to orange. Next $1 \mathrm{mmol}$ of tin precursor $\left(\mathrm{Ph}_{2} \mathrm{SnCl}_{2}(0.34 \mathrm{~g}) / \mathrm{Me}_{2} \mathrm{SnCl}_{2}(0.22 \mathrm{~g}) / \mathrm{SnCl}_{2}(0.19 \mathrm{~g})\right)$ was added to the mixture. The mixture was refluxed for 6 hours and filtered while hot to remove triethylamine salt and the filtrate was kept at room temperature until the product, an orange precipitate, formed.

Diphenyltin(IV) compound of 2-(2,3-dihydroxybenzylidene)-N-phenylhydrazine carbothioamide (14)

Orange solid. Yield: $71 \%$. Melting point: $133-137{ }^{\circ} \mathrm{C}$. Analysis calculated for $\mathrm{C}_{26} \mathrm{H}_{21} \mathrm{~N}_{3} \mathrm{O}_{2} \mathrm{SSn}: \mathrm{C}, 55.94 ; \mathrm{H}, 3.79 ; \mathrm{N}, 7.53 \%$. Found: C, 56.30; H, 3.99; N, 7.42\%. FT-IR $\left(\mathrm{ATR}, \mathrm{cm}^{-1}\right): 1587 v(\mathrm{C}=\mathrm{N}), 998 v(\mathrm{~N}-\mathrm{N}), 957 v(\mathrm{~S}-\mathrm{C}-\mathrm{S}) .{ }^{1} \mathrm{H}$ NMR (DMSO-d 6$) \delta(\mathrm{ppm})$ ): $6.57-$ 9.52 (m, 18H, Ar-H), 9.87 (s, 1H, CH), 11.69 (s, 1H, NH). ${ }^{13} \mathrm{C}$ NMR (DMSO-d 6 ) $\delta$ (ppm.): $120.5,120.7,125.4,125.5,125.6,125.7,128.5,128.6,128.7,128.8,128.9,129.0,129.1$, 129.5, 134.6, 135.2, 135.5, 136.3, 136.4, 136.7, 139.7 (Ar-C), $148.8(\mathrm{C}=\mathrm{N}), 175.2$ (S-C-S). ${ }^{119} \mathrm{Sn}$ NMR (DMSO-d 6 ) $\delta$ (ppm.): -328 .

Dimethyltin(IV) compound of 2-(2,3-dihydroxybenzylidene)-N-phenylhydrazine carbothioamide (15)

Orange solid. Yield: $42 \%$. Melting point: $153-156^{\circ} \mathrm{C}$. Analysis calculated for $\mathrm{C}_{16} \mathrm{H}_{17} \mathrm{~N}_{3} \mathrm{O}_{2} \mathrm{SSn}: \mathrm{C}, 44.27 ; \mathrm{H}, 3.95 ; \mathrm{N}, 9.68$. Found: C, 44.32; H, 3.72; N, 9.90\%. FT-IR $\left(\right.$ ATR, $\left.\mathrm{cm}^{-1}\right): 1575 v(\mathrm{C}=\mathrm{N}), 1001 v(\mathrm{~N}-\mathrm{N}), 917 v(\mathrm{~S}-\mathrm{C}-\mathrm{S}) .{ }^{1} \mathrm{H}$ NMR $\left(\mathrm{DMSO}-\mathrm{d}_{6}\right) \delta(\mathrm{ppm}): 0.63$ $\left(\mathrm{s}, 6 \mathrm{H}, \mathrm{CH}_{3}\right), 8.51(\mathrm{~s}, 1 \mathrm{H}, \mathrm{CH}), 6.33-7.75(\mathrm{~m}, 8 \mathrm{H}, \mathrm{Ar}-\mathrm{H}), 9.84(\mathrm{~s}, 1 \mathrm{H}, \mathrm{OH}) .{ }^{13} \mathrm{C} \mathrm{NMR}$ 
$\left(\mathrm{DMSO}_{-} \mathrm{d}_{6}\right) \delta$ (ppm.): $7.1\left(\mathrm{Sn}-\mathrm{CH}_{3}\right), 110.2,114.7,128.3,128.4,128.7,128.8,135.3,152.1$, 152.7, 153.6 (Ar-C), $153.8(\mathrm{C}=\mathrm{N}), 167.5(\mathrm{~S}-\mathrm{C}-\mathrm{S}) .{ }^{119} \mathrm{Sn}$ NMR $\left(\mathrm{DMSO}-\mathrm{d}_{6}\right) \delta(\mathrm{ppm}):-103$.

Tin(IV) compound of 2-(2,3-dihydroxybenzylidene)-N-phenylhydrazinecarbothioamide (16)

Compound 16 was prepared following the same procedure as described for 14, using 4 (0.58 g, 2 mmol). Orange solid. Yield: $48 \%$. Melting point: $>300{ }^{\circ} \mathrm{C}$. Analysis calculated for $\mathrm{C}_{28} \mathrm{H}_{22} \mathrm{~N}_{6} \mathrm{O}_{4} \mathrm{~S}_{2} \mathrm{Sn}: \mathrm{C}, 48.78 ; \mathrm{H}, 3.22 ; \mathrm{N}, 12.19 \%$. Found: C, 48.30; H, 2.97; N, $12.52 \%$. FTIR (ATR, $\left.\mathrm{cm}^{-1}\right): 1588 v(\mathrm{C}=\mathrm{N}), 1001 v(\mathrm{~N}-\mathrm{N}), 939 v(\mathrm{~S}-\mathrm{C}-\mathrm{S}) .{ }^{1} \mathrm{H}$ NMR (DMSO-d $) \delta$ (ppm.): 6.66-7.55 (m, 16H, Ar-H), 8.48 (s, 2H), 10.00 (s, 2H, CH), 11.74 (s, 2H, NH). ${ }^{13} \mathrm{C}$ NMR $\left(\right.$ DMSO-d $\left._{6}\right) \delta$ (ppm.): 117.1, 119.5, 121.2, 125.6, 125.7, 125.9, 126.0, 128.4, 128.5, 128.5, 139.6 (Ar-C), 145.9, $146.0(\mathrm{C}=\mathrm{N}), 175.7,176.1(\mathrm{~S}-\mathrm{C}-\mathrm{S}) .{ }^{119} \mathrm{Sn}$ NMR (DMSO-d 6$) \delta$ (ppm.): 541.

\subsection{X-ray Structure Determination}

Intensity data for light-yellow crystals of $\mathbf{1 1}^{\prime}(0.05 \times 0.08 \times 0.12 \mathrm{~mm})$ and $\mathbf{1 2}(0.07 \times 0.13 \times$ $0.18 \mathrm{~mm})$ a were measured at $150 \mathrm{~K}$ on an Oxford Diffraction Gemini Eos CCD diffractometer (Rigaku Oxford Diffraction, United Kingdom) fitted with Mo Ka radiation ( $\lambda$ $=0.71073 \AA$ ). Data reduction and empirical absorption corrections, based on a multi-scan technique, were applied [31]. The structures were solved by direct methods [32], and refined on $F^{2}$ with anisotropic displacement parameters and $\mathrm{C}$-bound $\mathrm{H}$ atoms in the riding model approximation [33]. The oxygen- and nitrogen-bound $\mathrm{H}$ atoms were refined with distance restraints of $\mathrm{O}-\mathrm{H}=0.84 \pm 0.01 \AA$ and $\mathrm{N}-\mathrm{H}=0.88 \pm 0.01 \AA$, respectively. A weighting scheme of the form $w=1 /\left[\sigma^{2}\left(F_{\mathrm{o}}{ }^{2}\right)+(a P)^{2}\right]$, where $P=\left(F_{\mathrm{o}}{ }^{2}+2{F_{\mathrm{c}}}^{2}\right) / 3$, was introduced in each case; for 11' $a=0.033$ and for $\mathbf{1 2} a=0.022$. The absolute structure of $\mathbf{1 2}$ was determined based on differences in Friedel pairs included in the data set. The molecular structure diagrams were generated at the $70 \%$ probability level by ORTEP for Windows [34], and the packing diagrams were prepared with DIAMOND [35]. Additional analysis was conducted with PLATON [36].

Crystal data for 11': $\mathrm{C}_{27} \mathrm{H}_{23} \mathrm{~N}_{3} \mathrm{O}_{2} \mathrm{SSn}, 0.5\left(\mathrm{C}_{16} \mathrm{H}_{16} \mathrm{~N}_{2} \mathrm{O}_{4}\right), M=722.43$, monoclinic, $P 2_{1} / c, a=$ 14.7977(5), $b=13.0726(4), c=17.0997(6) \AA, \beta=105.421(3)^{\circ}, V=3188.75(19) \AA^{3}, Z=4$, $D_{\mathrm{x}}=1.505 \mathrm{~g} \mathrm{~cm}^{-3}, F(000)=1468$, and $\mu=0.912 \mathrm{~mm}^{-1}$. No. reflections measured $=14861$ 
$\left(\theta_{\max }=29.4^{\circ}\right)$, no. independent reflections $=7337$, no. reflections with $I \geq 2 \sigma(I)=5378, R$ $($ obs. data $)=0.041$, and $w R_{2}($ all data $)=0.087$. CCDC deposition number: 1975499 .

Crystal data for 12: $\mathrm{C}_{17} \mathrm{H}_{19} \mathrm{~N}_{3} \mathrm{O}_{2} \mathrm{SSn}, M=448.10$, orthorhombic, $P 2{ }_{1} 2_{1}{ }_{1}, a=7.7253(2), b=$ 12.4692(3), $c=18.2692(5) \AA, V=1759.84(8) \AA^{3}, Z=4, D_{\mathrm{x}}=1.691 \mathrm{~g} \mathrm{~cm}^{-3}, F(000)=896$, and $\mu=1.585 \mathrm{~mm}^{-1}$. No. reflections measured $=11201\left(\theta_{\max }=29.3^{\circ}\right)$, no. independent reflections $=4191$, no. reflections $I \geq 2 \sigma(I)=3900, R$ (obs. data) $=0.030$, and $w R_{2}$ (all data) $=$ 0.096. CCDC deposition number: 1975500.

\subsection{Density Functional Theory (DFT) calculations}

DFT calculations were performed using Gaussian09 (Gaussian Inc., Wallingford, CT, USA) [37] and Gaussview5 (Semichem, Inc., Shawnee Mission, KS, USA) [38] software. The molecular structures and geometries of the Schiff bases and tin(IV) compounds were fully optimized using DFT method with the B3LYP $[39,40]$ hybrid exchange correlation functional with LanL2DZ pseudopotential on Sn [41-43] and 6-311G $(d, p)$ Pople basis set for all other atoms. The initial single crystal X-ray molecular structures and geometries for the tin compounds in $\mathbf{1 1}$ and $\mathbf{1 2}$ were used for DFT calculations using the same functional and basis set. Vibrational frequencies were scaled using a scaling factor of 0.9682 [44]. The electronic stabilities of the optimized geometries were computed using the time-dependent density functional theory (TD-DFT) formalism [45,46] and included solvation effects (DMSO) via the polarizable continuum method (PCM) [47-49], using the same basis set. These DFT were performed in the same way as reported in a previous publication [25].

\subsection{In Vitro Cytotoxic Assay}

The cytotoxicity of tin(IV) compounds against HT29 (colon), U87 and SJ-G2 (glioblastoma), MCF-7 (breast), A2780 (ovarian), H460 (lung), A431 (skin), DU145 (prostate), BE2-C (neuroblastoma), and MIA (pancreas) cell lines and one normal breast cell line, MCF-10A were performed by the MTT assay using the same method as previously reported [25,50,51].

Cell Culture and Stock Solutions. Stock solutions were prepared as follows and stored at -20 ${ }^{\circ} \mathrm{C}$ : Trial compounds were stored as $10 \mathrm{mM}$ solutions in DMSO. All cell lines were cultured in a humidified atmosphere $5 \% \mathrm{CO}_{2}$ at $37{ }^{\circ} \mathrm{C}$. The cancer cell lines were maintained in 


\section{Results and discussion}

\subsection{Synthesis}

The synthetic pathway of the Schiff bases (1-4) and their tin(IV) compounds (5-16) are indicated in Schemes 1 and 2. The Schiff bases were synthesized by the condensation reaction between 2-hydroxy-3-methoxybenzaldehyde/ 2,3-dihydroxybenzaldehyde and the corresponding thiosemicarbazide (4-methyl-3-thiosemicarbazide and 4-phenyl-3thiosemicarbazide) in alcoholic solution, which was as previously reported [27,54,55]. The Schiff bases were then reacted with $\mathrm{Ph}_{2} \mathrm{SnCl}_{2}, \mathrm{Me}_{2} \mathrm{SnCl}_{2}$ and $\mathrm{SnCl}_{2}$ separately, in the presence of potassium hydroxide $(\mathrm{KOH}) /$ triethyamine $\left(\mathrm{Et}_{3} \mathrm{~N}\right)$ by conventional methods or under reflux. The isolated yellow or orange colored tin(IV) compounds were achieved in acceptable yields (31-79\%), however, some were produced in low yields due to their 
instability at room temperature. The tin(IV) compounds were soluble in most organic solvents especially dimethylsulfoxide (DMSO) and dimethylformamide (DMF). The molar conductance values of the compounds were in the range $0.88-7.85 \Omega^{-1} \mathrm{~cm}^{2} \mathrm{~mol}^{-1}$, which was well below than $25 \Omega^{-1} \mathrm{~cm}^{2} \mathrm{~mol}^{-1}$ indicating that all of them were non-electrolytic in nature. This meant that no counter ions were present in the outer coordination sphere [56].<smiles>[R7]NC(=S)N/N=C/c1cccc([R2])c1O</smiles>

Scheme 1. Synthetic pathway to thiosemicarbazone Schiff bases 1-4.

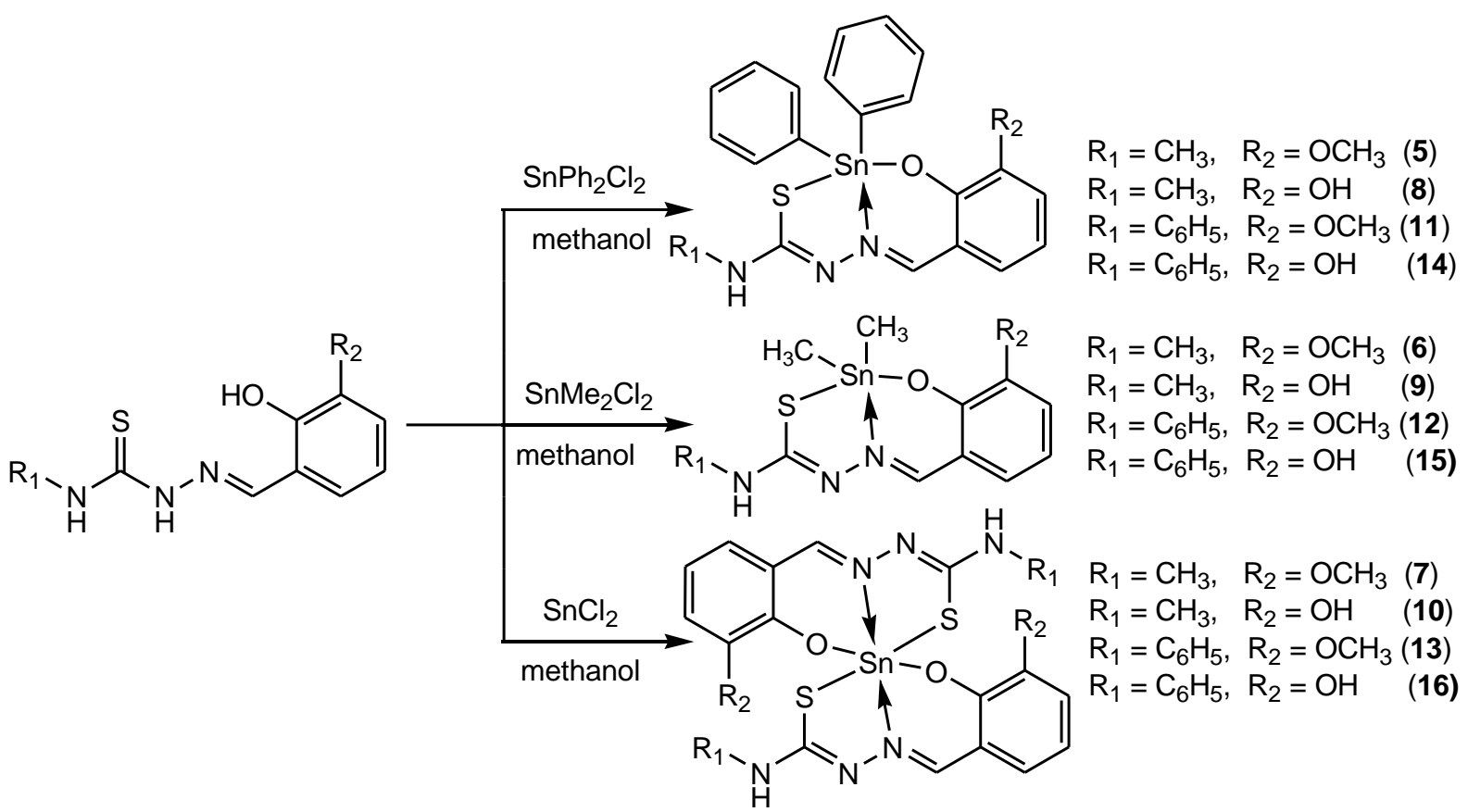

Scheme 2. Synthetic pathway of thiosemicarbazone Schiff bases to tin(IV) compounds (5-16)

\section{2. $\quad$ Spectroscopic and spectrometric data}

\subsubsection{FTIR analysis}


The experimental and calculated frequencies in the infrared spectra of thiosemicarbazone Schiff bases (1-4) and their tin(IV) compounds (5-16) were determined in the range of 4000$280 \mathrm{~cm}^{-1}$ and $4000-0 \mathrm{~cm}^{-1}$, respectively. Important infrared vibrations and their assignments are summarized in Table S1 for both experimental and calculated frequencies. The calculated frequencies were employed to assign prominent peaks with maximum accuracy, which resulted in excellent correlation with experimental data (Figure S1). In the spectra of $\mathbf{1}$ and $\mathbf{2}$, the $v(\mathrm{OH})$ was not observed, which suggested that the $v(\mathrm{OH})$ band overlapped with the $v(\mathrm{~N}-$ $\mathrm{H})$ band due to hydrogen bonding $(\mathrm{NH} . . . \mathrm{OH})$ between the two groups [57,58]. The $v(\mathrm{OH})$ was observed in the calculated spectra because they were generated from the gas phase structures, while experimental spectra were analyzed in the solid state where the compounds are in a more concentrated form, resulting in either intermolecular and/or intramolecular hydrogen bonding similar to that observed in structurally-related Schiff bases $[23,59,60]$. Conversely, $v(\mathrm{OH})$ was observed in the spectra of $\mathbf{3}$ and $\mathbf{4}$ which were comparable to previous literature [23]. As a result, the loss of $v(\mathrm{OH})$ upon complexation was difficult to assign by FTIR alone, due to the intra- and intermolecular hydrogen bonding between the molecules. The $v(\mathrm{~N}-\mathrm{H})$ of thiosemicarbazone Schiff bases disappeared upon complexation due to the deprotonation of $\mathrm{NH}$ and the involvement of resulting nitrogen atom in coordination to the Sn center. Furthermore, the IR spectra of 1, 2, 3 and 4 exhibited a strong intensity band due to the presence of $v(\mathrm{C}=\mathrm{N})_{\text {azomethine }}$ at 1610, 1609, 1601 and $1597 \mathrm{~cm}^{-1}$, respectively. This band shifted to lower frequencies in the spectra of tin(IV) compounds suggesting coordination to the tin center via the azomethine nitrogen atom. Other than that, the $v(\mathrm{C}=\mathrm{S})$ and $v(\mathrm{~N}-\mathrm{N})$ absorptions shifted to lower frequencies upon complexation, indicating coordination via thiolate sulfur and azomethine nitrogen forming five-membered chelate rings. A small deviation was observed in the vibrational frequencies which can be explained by the fact that the experimental spectra was obtained in the solid state, while DFT calculations were run in the gas phase.

\subsubsection{Multinuclear $\left({ }^{1} \mathrm{H}-,{ }^{13} \mathrm{C}\right.$ - and ${ }^{119} \mathrm{Sn}$-) $\mathrm{NMR}$ spectral analysis}

The ${ }^{1} \mathrm{H}$ - and ${ }^{13} \mathrm{C}$ - NMR spectra of 1-4 were recorded in DMSO- $\mathrm{d}_{6}$ solution and 5-16 were recorded in DMSO- $\mathrm{d}_{6} / \mathrm{CDCl}_{3}$ solution at room temperature. The assignments of the relevant signals are compiled in Tables S2 and S3. The ${ }^{1} \mathrm{H}-\mathrm{NMR}$ spectra of 1-4 showed signals at $11.42,11.78,11.40$ and $11.75 \mathrm{ppm}$, respectively, which indicated the presence of -NHprotons. These -NH- proton signals were not present in the spectra of the tin(IV) compounds indicating that the Schiff bases were coordinated to the tin atoms via the nitrogen donor atom. 
Proton signals appeared at 9.18 and 10.02 ppm for $\mathbf{1}$ and $\mathbf{2}$ corresponding to the hydroxyl coordination of the hydroxyl proton to the tin center [61]. Contrastingly, the two signals for hydroxyl groups at 8.37, $8.38 \mathrm{ppm}$ (3) and 8.96, $9.52 \mathrm{ppm}$ (4) disappeared upon complexation, which indicated the presence of intra- and intermolecular hydrogen bonding in the compounds $[23,62]$.

The ${ }^{13} \mathrm{C}$ - NMR spectra of $\mathbf{1}, \mathbf{2}, \mathbf{3}$, and $\mathbf{4}$ showed carbon signals at 177.6, 176.1, 178.0, and $176.0 \mathrm{ppm}$, respectively at the downfield region attributed to $-\mathrm{S}-\mathrm{C}(=\mathrm{S}) \mathrm{N}$. The position of these carbon signals proved that the compounds are predominately thione tautomers even in DMSO- $\mathrm{d}_{6}$ solution. The signals shifted to the upfield region in the spectra of tin(IV) compounds indicative of the involvement of $-\mathrm{S}-\mathrm{C}(-\mathrm{S}) \mathrm{N}$ in the complexation, and decreasing electron density at the carbon atom when sulfur was chelated to the tin atom. The $\mathrm{C}=\mathrm{N}$ signal was assigned at 148.4, 148.6, 146.0, and $146.0 \mathrm{ppm}$ for 1-4, respectively, and appeared downfield as the carbon atom was bonded to the electronegative atoms. However, the $\mathrm{C}=\mathrm{N}$ signals shifted downfield in the spectra of tin(IV) compounds due to the increasing electron

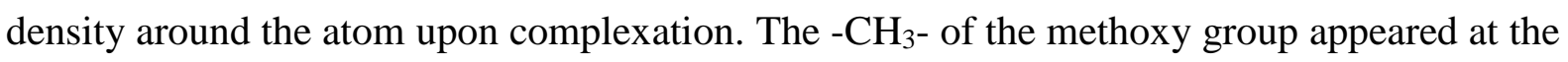
upfield region at 56.4 (1) and $56.3 \mathrm{ppm}$ (2). A similar carbon signal was observed for the $\mathrm{CH}_{3}$ - of the methoxy group in the spectra of tin(IV) compounds indicating that the methoxy group did not coordinate to the tin atom.

${ }^{119} \mathrm{Sn}$ - NMR was used to predict the geometry of the tin-containing compounds. The ${ }^{119} \mathrm{Sn}$ NMR spectra of compounds 5-16 were evaluated in $\mathrm{DMSO} / \mathrm{CDCl}_{3}$ solutions, at room temperature using $\mathrm{SnCl}_{4}(\delta=-150 \mathrm{ppm})$ as an external standard. The ${ }^{119} \mathrm{Sn}$ chemical shift strongly depends on the alkyl/aryl group attached to the tin atom and the electronegativity of the ligand coordinated to the tin atom as well as temperature employed in the experiments. Theoretically, as the coordination number increases, the ${ }^{119} \mathrm{Sn}$ chemical shift moves towards the shielding region [63]. The spectra showed one sharp signal which indicated that the tin(IV) compounds had only a single tin atom species. The ${ }^{119} \mathrm{Sn}$ NMR values of pentacoordinated diphenyl- $(\mathbf{5}, \mathbf{8}, \mathbf{1 1}$, and 14) and dimethyltin(IV) $(\mathbf{6}, \mathbf{9}, \mathbf{1 2}$, and 15) compounds fell in the range of -227 to -328 and -103 to $-154 \mathrm{ppm}$, respectively, similar to that reported previously for diphenyl- and dimethyltin(IV) compounds [64-67]. The ${ }^{119} \mathrm{Sn}$ NMR values of hexa-coordinated tin(IV) compounds $(\mathbf{7}, 10,13$, and 16) were observed in the range of -354 to $-541 \mathrm{ppm}$. The ${ }^{119} \mathrm{Sn}-\mathrm{NMR}$ values of compounds $\mathbf{8}, \mathbf{1 0}, \mathbf{1 4}$, and 16 were more negative due 
to the presence of hydroxyl groups at the meta position, which were more electronegative than the methoxy groups [68].

\subsubsection{Mass spectrometric analysis}

Mass spectral data for 1-4 were recorded in DMSO and were found to be consistent with the proposed formulation of the Schiff bases. The mass spectra displayed prominent peaks at $\mathrm{m} / \mathrm{z}$ 239, 301, 225, and 287 for Schiff bases 1-4, respectively, which correspond to the $\left[\mathrm{C}_{10} \mathrm{H}_{13} \mathrm{~N}_{3} \mathrm{O}_{2} \mathrm{~S}\right]^{+},\left[\mathrm{C}_{15} \mathrm{H}_{15} \mathrm{~N}_{3} \mathrm{O}_{2} \mathrm{~S}\right]^{+},\left[\mathrm{C}_{9} \mathrm{H}_{11} \mathrm{~N}_{3} \mathrm{O}_{2} \mathrm{~S}\right]^{+}$and $\left[\mathrm{C}_{14} \mathrm{H}_{13} \mathrm{~N}_{3} \mathrm{O}_{2} \mathrm{~S}\right]^{+}$ions; the mass spectra for 1-4 are supplied in Figure S2.

\subsubsection{Electronic spectral analysis}

The experimental and calculated electronic data of compounds 1-16 in DMSO are tabulated in Table S4. The prominent experimental electronic absorptions for 1-4 were observed in the range of 328-334 $\mathrm{nm}$, which were best correlated with the calculated absorptions by B3LYP in the range of 317-330 nm. The frontier molecular orbitals of compounds 1-16 are shown in Figure S3, where the figure illustrated the excitation of electrons from HOMO of nonbonding electrons at sulfur and nitrogen atoms that were excited to the LUMO which was largely centered on the thiosemicarbazone backbone, 2,3-dihydroxy phenyl ring and as well as the oxygen atom attached to the phenyl ring. Thus, this supported the transition of electrons from $n \rightarrow \pi^{*}$ and $\pi \rightarrow \pi^{*}$ of the Schiff bases. For tin(IV) compounds (5-16), the HOMOs were largely centered on the thiosemicarbazone Schiff base, whereas the LUMOs were centered on the entire thiosemicarbazone Schiff base except the methyl or phenyl groups attached to the nitrogen atom, thiolate sulfur and oxygen atoms attached to the phenyl ring.

\subsection{X-ray structure crystallography of 11' and 12}

\subsubsection{Molecular structures}

The crystallographic asymmetric unit of 11' comprises a molecule of $\mathrm{Ph}_{2} \mathrm{Sn}\left(\mathrm{L}^{2}\right)(\mathbf{1 1})$ and half a molecule of 3-methoxysalicylaldehyde azine with the molecular structures of each shown in Figure 2. The presence of the azine molecule in $\mathbf{1 1}^{\prime}$ presumably arises from the prolonged standing of the acetonitrile:methanol (1:1) solution during crystallization of an authenticated sample of $\mathbf{1 1}$ which resulted in partial decomposition of $\mathbf{1 1}$ and subsequent condensation of hydrazine and $o$-vanillin to form the azine. The tin center in $\mathbf{1 1}$ ' is coordinated by two ipso- 
(a)
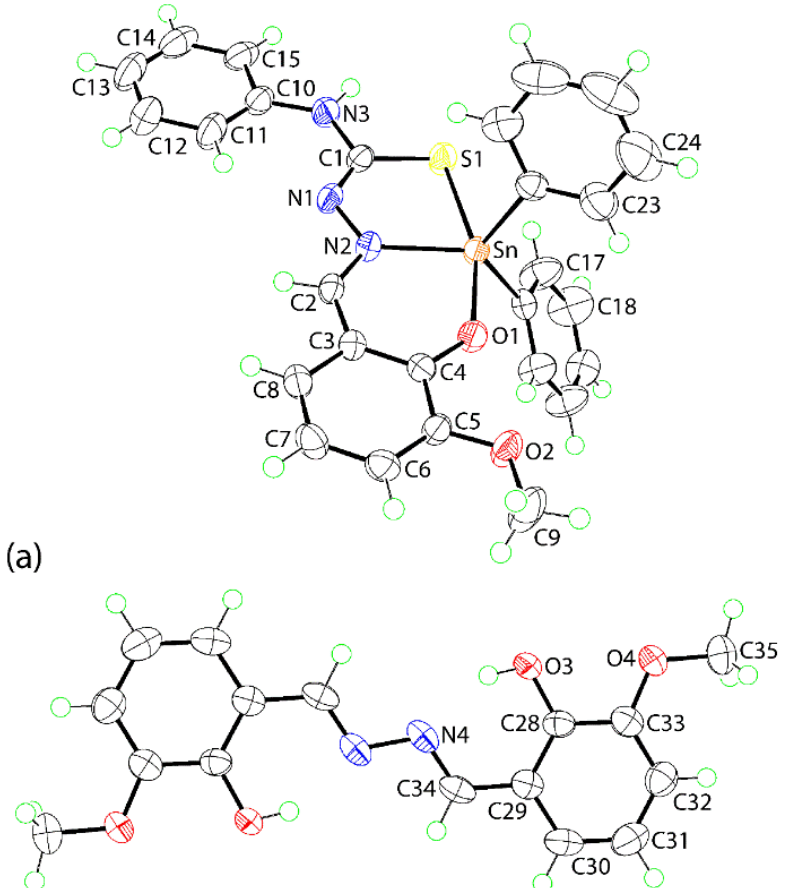

(b)

Figure 2. The molecular structure of the constituents of 11' showing atom-labeling scheme and $70 \%$ probability displacement ellipsoids. The 3-methoxysalicylaldehyde azine molecule in (b) is disposed about a crystallographic center of inversion with unlabeled atoms related by the symmetry operation $-x, 3-y,-z$. Selected geometric parameters: Sn-S1 $=2.5475(8), \mathrm{Sn}-$ 
$\mathrm{O} 1=2.0853(19), \mathrm{Sn}-\mathrm{N} 2=2.176(3), \mathrm{S} 1-\mathrm{C} 1=1.748(3), \mathrm{N} 1-\mathrm{N} 2=1.394(3), \mathrm{N} 1-\mathrm{C} 1=$

To a first approximation, the molecular structure of 12, Figure 3, mirrors that found for the diphenyltin compound in 11'. Thus, a similar coordination mode is adopted by the $\mathrm{L}^{1}$ dianion but, in this case, based on a value of $\tau=0.0$ [69], the coordination geometry is distorted trigonal-bipyramidal. In this description, the Sn atom lies 0.6358(18) $\AA$ out of the basal plane defined by the S1, O1, N2 and C16 atoms [r.m.s. deviation $=0.0078 \AA$ ] in the direction of the axially-bound $\mathrm{C} 17$ atom. This arises as the two widest angles, that is, S1-Sn-O1 $\left[145.67(9)^{\circ}\right]$ and N2-Sn-C16 $\left[145.45(15)^{\circ}\right]$ are virtually identical. The Sn-S1 [2.5475(8)] and Sn-N2 [2.257(3)] bond lengths are, respectively, approximately $0.05 \AA$ shorter and 0.08 $\AA$ longer in 12 than the equivalent bonds in 11', while the Sn-O1 bond lengths remain the same. 
Figure 3. The molecular structure of the constituents of $\mathbf{1 2}$ showing atom-labeling scheme and $70 \%$ probability displacement ellipsoids. Selected interatomic parameters: $\mathrm{Sn}-\mathrm{S} 1=$ 2.4982(12), Sn-O1 2.085(3), Sn-N2 = 2.257(3), S1-C1 = 1.751(4), N1-N2 = 1.396(5), N1$\mathrm{C} 1=1.306(5)$, and N2-C2 $=1.288(5) \AA$.

The five- and six-membered chelate rings in $\mathbf{1 2}$ exhibited r.m.s. deviations of $0.080 \AA$ [maximum deviation $=0.071(3) \AA$ for the $\mathrm{N} 2$ atom and $0.200 \AA$ [0.189(1) $\AA$ for Sn], suggesting deviations from planarity. Indeed, the five- and six-membered rings may each be described as having an envelope conformation where, for the smaller ring, the Sn atom lies $0.248(6) \AA$ out of the plane defined by the remaining four atoms [r.m.s. deviation $=0.0056$ $\AA$ ]. The envelope is more pronounced for the larger ring with the Sn atom 0.612(5) $\AA$ above the plane [r.m.s. deviation $=0.0229 \AA$ ]. The dihedral angle between the chelate rings is $17.88(12)^{\circ}$ but this reduces to $12.1(2)^{\circ}$ when the angle between the planar regions is computed. The dihedral angle between the outer rings is $6.2(2)^{\circ}$.

Thus far, no specific mention of the tin-bound substituents in $\mathbf{1 1}^{\prime}$ and $\mathbf{1 2}$ has been made. The Sn-C bond lengths in 11' are equivalent at $2 \times 2.134(3)$ and, in turn, these are experimentally equivalent to those in 12, that is, 2.134(4) $\AA$ [Sn-C16] and 2.128(4) $\AA$ [Sn-C17]. A difference is seen in the $\mathrm{C}-\mathrm{Sn}-\mathrm{C}$ angles, however. Thus, in $\mathbf{1 1}^{\prime}$, this angle is $121.46(12)^{\circ}$ which is significantly wider than the equivalent angle of $114.82(18)^{\circ}$ in $\mathbf{1 2}$. This disparity is emphasized in the overlay diagram shown in Figure 4, as are the differences in the relative orientations of the $\mathrm{L}^{2}$ di-anions. 


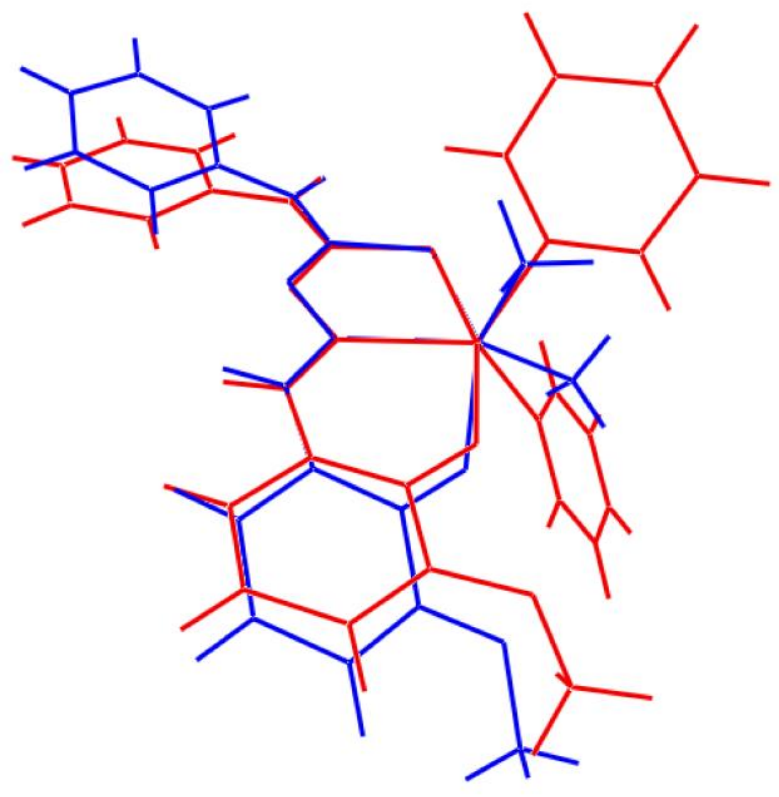

Figure 4. Overlay diagram of the $\mathrm{R}_{2} \mathrm{Sn}\left(\mathrm{L}^{2}\right)$ molecules in $\mathbf{1 1}^{\prime}(\mathrm{R}=\mathrm{Ph}$; red image) and inverted-12 ( $\mathrm{R}=\mathrm{Me}$; blue image) whereby the five-membered rings are coincident.

\subsubsection{Supramolecular structures}

The most notable aspect of the molecular packing of $\mathbf{1 1}^{\prime}$ is the formation of eight-membered $\{\cdots \mathrm{HNCS}\}_{2}$ synthons through the agency of amine- $\mathrm{N}-\mathrm{H}^{\cdots} \mathrm{S}$ (thiolate) hydrogen bonds between centrosymmetrically related $\mathrm{Ph}_{2} \mathrm{Sn}\left(\mathrm{L}^{2}\right)$ molecules, Figure 5(a). Additional interactions between molecules of note are of the type $\pi$ (chelate ring) ${ }^{\cdots} \pi$ (oxidobenzylidene) stacking as illustrated in Figure 5(b). Such interactions are increasingly being recognized as being important in providing points of contact in coordination chemistry [73] and computational chemistry indicates these provide energies of stabilization greater than conventional $\pi$ stacking interactions between organic residues [74]. The dimeric aggregates are connected into a supramolecular layer in the $a b$-plane via $\mathrm{L}^{1}$-imine- $\mathrm{C}-\mathrm{H} \cdots \mathrm{O}$ (hydroxy) and imine-C$\mathrm{H}^{\cdots} \mathrm{O}$ (methoxy) interactions as shown in Figure 5(c). In essence, each 3methoxysalicylaldehyde azine molecule links four symmetry related $\mathrm{Ph}_{2} \mathrm{Sn}\left(\mathrm{L}^{2}\right)$ molecules. The layers stack along the $c$-axis direction, being connected by tin-bound-phenyl-C- $\mathrm{H}^{\cdots} \pi(\mathrm{Sn}-$ phenyl, oxidobenzylidene) and azine-methoxy-C-H ${ }^{\cdots} \pi(\mathrm{N}$-phenyl) interactions to consolidate the three-dimensional architecture, Figure 5(d). 
(a)

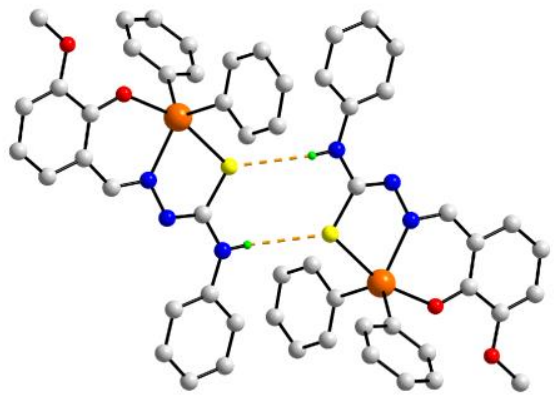

(c)
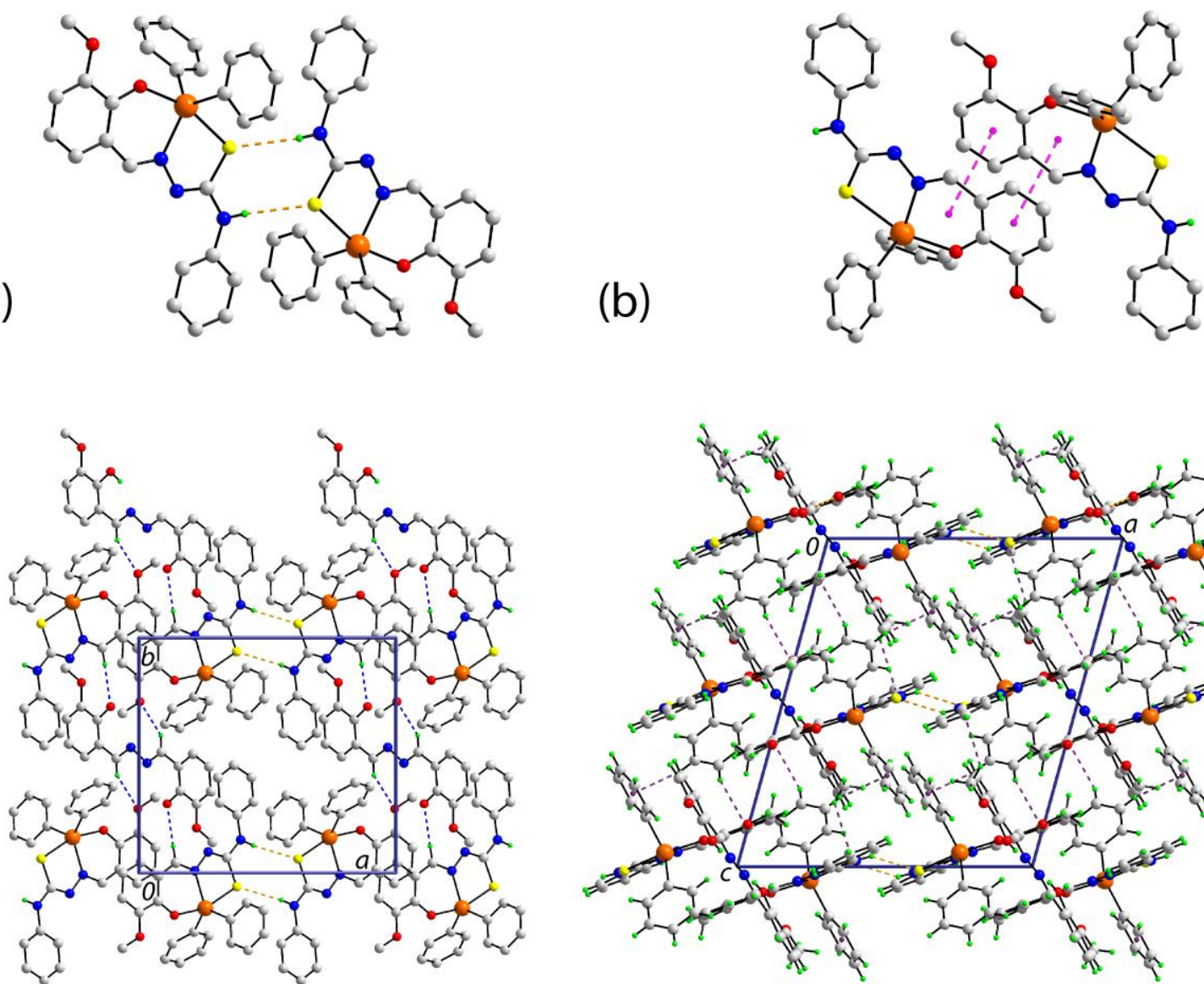

(b)

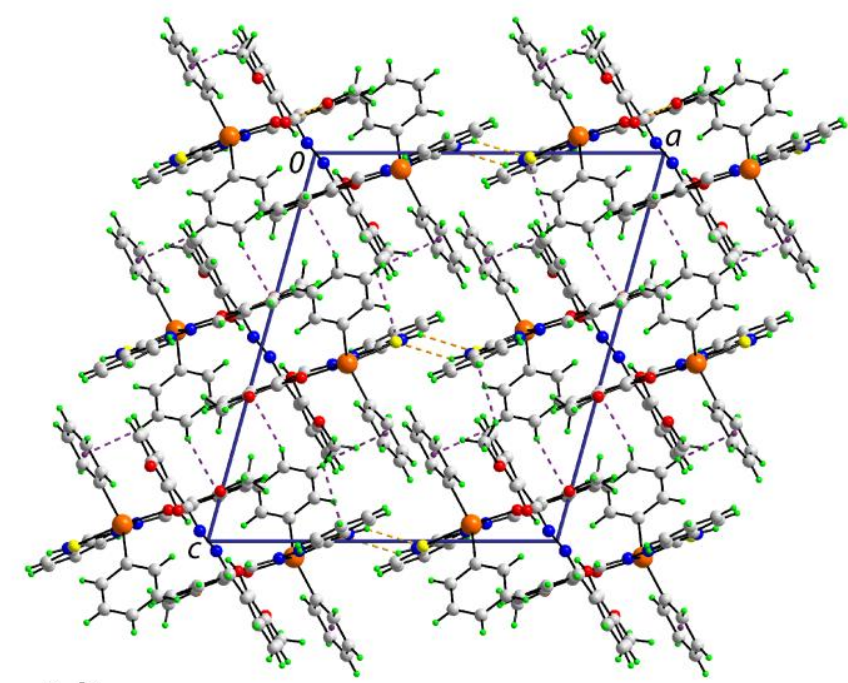

(d)

Figure 5. Molecular packing in the crystal of 11': (a) a view of the supramolecular dimer sustained by amine- $\mathrm{N}-\mathrm{H}^{\cdots} \mathrm{S}$ (thiolate) hydrogen bonds, shown as orange dashed lines [N3$\mathrm{H} 3 \mathrm{n}^{\cdots} \mathrm{S}^{\mathrm{i}}{ }^{\mathrm{i}}: \mathrm{H} 3 \mathrm{n}^{\cdots} \mathrm{S} 1^{\mathrm{i}}=2.60(3) \AA, \mathrm{N} 3{ }^{\cdots} \mathrm{S} 1^{\mathrm{i}}=3.380(3) \AA$ and angle at $\mathrm{H} 3 \mathrm{n}=150(3)^{\circ}$ for symmetry operation (i) $1-x, 2-y,-z]$, (b) a view of the dimer aggregate connected by $\pi$ (chelate ring) ${ }^{\cdots} \pi$ (oxidobenzylidene) stacking interactions $\left[\mathrm{Cg}(\mathrm{Sn}, \mathrm{O} 1, \mathrm{~N} 2, \mathrm{C} 1-\mathrm{C} 3) \ldots \mathrm{Cg}(\mathrm{C} 3-\mathrm{C} 8)^{\mathrm{ii}}=\right.$ $3.8613(15) \AA$ and angle of inclination $=2.13(11)^{\circ}$ for (ii) $\left.-x, 2-y,-z\right]$ shown as pink dashed lines, (c) supramolecular layer whereby the aggregate shown in (a) is connected by $\mathrm{L}^{2}$-imine$\mathrm{C}-\mathrm{H}^{\cdots} \mathrm{O}$ (hydroxy) and imine- $\mathrm{C}-\mathrm{H}^{\cdots} \mathrm{O}$ (methoxy) interactions (blue dashed lines) [C2$\mathrm{H} 2 \cdots \mathrm{O} 3: \mathrm{H} 2 \cdots \mathrm{O} 3=2.40 \AA, \mathrm{C} 2 \cdots \mathrm{O} 3=3.336(4) \AA$ and angle at $\mathrm{H} 2=168^{\circ} ; \mathrm{C} 34-\mathrm{H} 34 \cdots \mathrm{O} 2^{\mathrm{iii}}$ : $\mathrm{C} 34-\mathrm{H} 34 \cdots \mathrm{O} 2^{\mathrm{iii}}=2.40 \AA, \mathrm{C} 34 \cdots \mathrm{O} 2^{\mathrm{iii}}=3.230(4) \AA$ and angle at $\mathrm{H} 34=146^{\circ}$ for (iii) $\left.x, 1+y, z\right]$, and (d) a view of the unit-cell contents in projection down the $b$-axis with $\mathrm{C}-\mathrm{H}^{\cdots} \pi$ interactions shown as purple dashed lines $\left[\mathrm{C} 18-\mathrm{H} 18 \cdots \mathrm{Cg}(\mathrm{C} 22-\mathrm{C} 27)^{\mathrm{iv}}: \mathrm{H} 18 \cdots \mathrm{Cg}(\mathrm{C} 22-\mathrm{C} 27)^{\mathrm{iv}}=\right.$ $2.79 \AA$ and angle at $\mathrm{H} 18=148^{\circ} ; \mathrm{C} 19-\mathrm{H} 19 \cdots \mathrm{Cg}(\mathrm{C} 3-\mathrm{C} 8)^{\mathrm{iv}}: \mathrm{H} 19 \cdots \mathrm{Cg}(\mathrm{C} 3-\mathrm{C} 8)^{\mathrm{iv}}=2.73 \AA$ and angle at $\mathrm{H} 19=138^{\circ} ; \mathrm{C} 35-\mathrm{H} 35 \mathrm{c}^{\cdots} \mathrm{Cg}(\mathrm{C} 10-\mathrm{C} 15)^{\mathrm{v}}: \mathrm{H} 35 \mathrm{c}^{\cdots} \mathrm{Cg}(\mathrm{C} 10-\mathrm{C} 15)^{\mathrm{v}}=2.85 \AA$ and angle at 
$\mathrm{H} 35 \mathrm{c}=133^{\circ}$ for (iv) $x, 3 / 2-y,-1 / 2+z$ and (v) $\left.x, 5 / 2-y, 1 / 2+z\right]$. In (a)-(c), non-participating hydrogen atoms have been omitted for clarity.

The most prominent aspect of the molecular packing of $\mathbf{1 2}$ is the formation of supramolecular chains supported by amine- $\mathrm{N}-\mathrm{H} \cdots \mathrm{O}$ (phenoxide) hydrogen bonding. The chains are aligned along the $a$-axis and have helical topology, being propagated by $2{ }_{1}$-screw symmetry, Figure 6(a). Further stability to the aforementioned chains is provided by secondary bonding $[73,75]$ of the type $\mathrm{Sn}^{\cdots} \mathrm{S}$, well known in organotin chemistry [76]. As detailed in Figure 6(b), the sulfur atom approaches the tin atom from the basal plane to establish a $5+1$ coordination geometry; the $\mathrm{C} 17-\mathrm{Sn}-\mathrm{S} 1$ angle $=157.48(11)^{\circ}$. When considered in conjunction with the hydrogen bonding, six-membered, $\{\cdots \mathrm{HNCS} \cdots \mathrm{SnO}\}$ heterosynthons are established. The chains are assembled into a three-dimensional architecture by amine-N-phenyl-C$\mathrm{H}^{\cdots} \pi$ (oxidobenzylidene) interactions as each chain forms two donor and two acceptor interactions, Figure 6(c). 
(a)
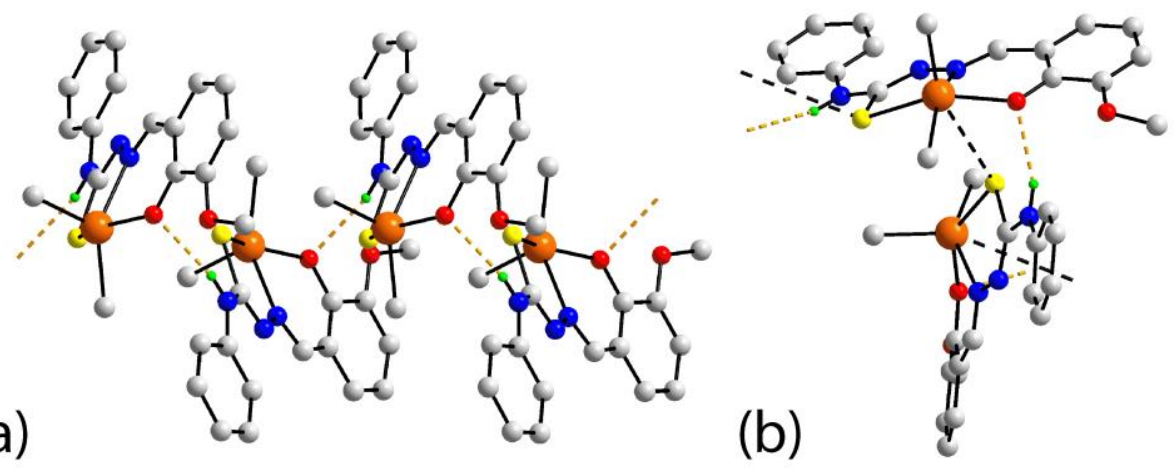

(b)

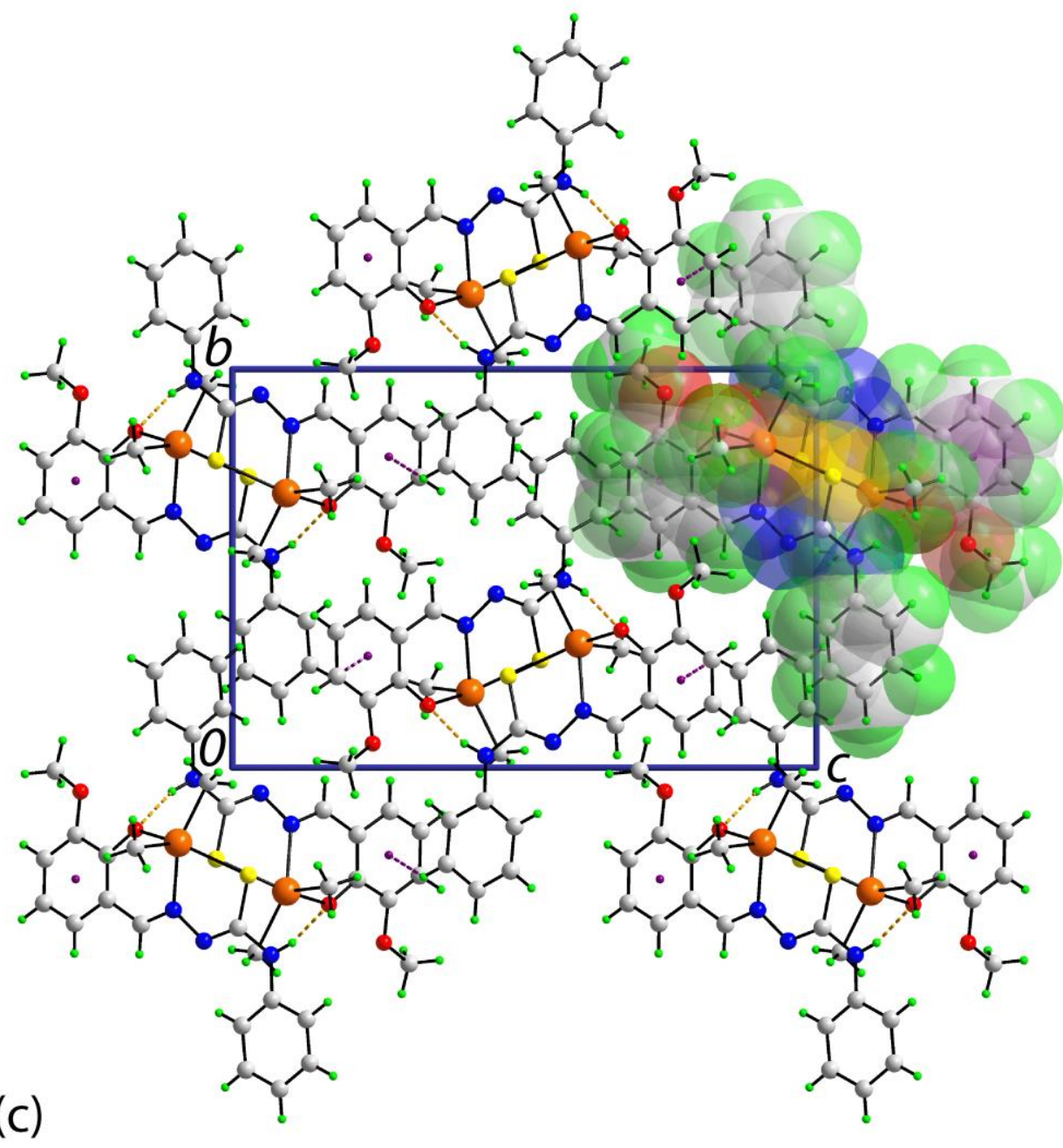

Figure 6. Molecular packing in the crystal of 12: (a) a view of the supramolecular helical chain sustained by amine- $\mathrm{N}-\mathrm{H}^{\cdots} \mathrm{O}$ (phenoxide) hydrogen bonds, shown as orange dashed lines $\left[\mathrm{N} 3-\mathrm{H} 3 \mathrm{n}^{\cdots} \mathrm{O} 1^{\mathrm{i}}: \mathrm{H} 3 \mathrm{n}^{\cdots} \mathrm{O} 1^{\mathrm{i}}=2.26(4) \AA, \mathrm{N} 3 \cdots \mathrm{O} 1^{\mathrm{i}}=3.089(5) \AA\right.$ and angle at $\mathrm{H} 3 \mathrm{n}=160(3)^{\circ}$ for symmetry operation (i) $1 / 2+x, 1 / 2-y, 1-z]$, (b) detail of the $\mathrm{Sn} \cdots \mathrm{S}$ secondary bonding [Sn $\cdots \mathrm{S} 1^{\mathrm{ii}}$ $=3.4928(12) \AA$ for (ii) $-1 / 2+x, 1 / 2-y, 1-z]$ within the chain shown in (a) and the resulting sixmembered, $\{\cdots \mathrm{HNCS} \cdots \mathrm{SnO}\}$ heterosynthon, and (c) a view of the unit-cell contents in projection down the $a$-axis with one supramolecular chain highlighted in space-filling mode. 
Chains are connected by amine-N-phenyl-C $-\mathrm{H}^{\cdots} \pi$ (oxidobenzylidene) interactions [C12$\mathrm{H} 12 \cdots \mathrm{Cg}(\mathrm{C} 3-\mathrm{C} 8)^{\mathrm{iii}}: \mathrm{H} 12 \cdots \mathrm{Cg} 3^{\mathrm{iii}}=2.98 \AA$ and angle at $\mathrm{H} 12=129^{\circ}$ for (iii) $\left.1 / 2-x, 1-y, 1 / 2+z\right]$ shown as purple dashed lines.

\subsection{Cytotoxic activity}

Compounds 1-16 were screened for their cytotoxicity against a panel of ten cancer cell lines, HT29, U87, SJ-G2, MCF-7, A2780, H460, A431, DU145, BE2-C, and MIA cell lines and one normal cell line, MCF-10A (Table 1). However, it was not possible to determine the cytotoxicity values of 7 due to its insolubility in $100 \%$ DMSO at $1 \mathrm{mM}$ concentration. Cisplatin was used as a positive control to induce cell death. The growth inhibition concentration of the compounds required to inhibit $50 \%$ cell proliferation $\left(\mathrm{GI}_{50}\right)$ were recorded after 72 hours of cell exposure to the compounds. The stability of the compounds in DMSO and in a mixture of DMSO and $\mathrm{H}_{2} \mathrm{O}$ were studied by UV-vis spectroscopic analysis, where the spectra remained unchanged after 72 hours which indicated that the compounds were stable in both solvent systems.

The cytotoxicity evaluation of the 2-hydroxy-3-methoxybenzyl thiosemicarbazone Schiff base analogues (1 and 2) revealed an increase in potency when a methyl substituent was attached to the $\alpha$-nitrogen atom, where 1 exhibited 10 to 20 times higher anti-proliferative activity as compared that of $\mathbf{2 , 3}$, and $\mathbf{4}$ in the panel of cancer cell lines tested. Table 1 shows the high level of cytotoxic potency of 1 against HT-29, A2780, A431, BE2-C, and MIA cell lines. Compound 1 was approximately $\sim 10-100$ times more potent than similar synthesized structures, 2-[(1E)-(\{[(Benzylsulfanyl)methanethioyl $]$ amino $\}$-imino $)$ methyl $]-6-$ methoxyphenol (SBOVaH) [25] and 2-hydroxy-5-methoxybenzaldehyde-N(4)methylthiosemicarbazone (H2dmmt) [8] against all the cancer cell lines tested, except for the DU145 cell line. 1 also showed excellent cytotoxicity against the panel of cancer cell lines compared to the reference drug (cisplatin). No obvious cytotoxicity pattern was observed for 2 which was similar to that of a similar analogue, SBOVaH [25]. In contrast, the 2,3dihydroxybenzyl thiosemicarbazone Schiff bases (3 and 4) showed a different pattern of cytotoxicity, which could be attributed to the phenyl group attached to the $\alpha$-nitrogen, where the phenyl group potentially facilitates the binding to biological molecules by $\pi$ interactions [23]. Compounds $\mathbf{2}$ and $\mathbf{4}$ were similar in structure, with the difference only in the methoxy (2) and hydroxyl (4) group substituents at the meta position of benzene rings. Compound 4 
was more active than $\mathbf{2}$ in all cancer cell lines tested. This was possibly due to the formation of hydrogen bonding interactions of two hydroxyl groups with the active site of amino acids of various enzymes in cancer cells [77]. Compound $\mathbf{3}$ showed poorer cytotoxicity at the 25 $\mu \mathrm{M}$ single point dose evaluation pre-screening and was not selected for further $\mathrm{GI}_{50}$ determination, as it was considered to be inactive. The cytotoxicity of the Schiff bases was tested using the non-cancerous normal human breast cell line (MCF10A) where 1 showed lower toxicity towards the normal cells, which was evident from its higher GI50 values (less active) as compared to the $\mathrm{GI}_{50}$ values of most of the cancer cells, except U87, H460 and DU145. Compound 4 also exhibited higher GI $_{50}$ values against MCF10A than HT29, MCF-7 and A2780. This suggested that $\mathbf{1}$ and $\mathbf{4}$ exhibited notable anticancer properties against certain cancer cells as compared to normal cells.

The cytotoxicity of the tin(IV) compounds are comparable to that of related compounds [25] where the diphenyltin(IV) compounds exhibited higher activities against certain cell lines as compared to their Schiff bases and other tin(IV) compounds. In particular, 5 showed higher cytotoxicity than its Schiff base (1) towards MCF-7, A2780, H460 and DU145 cells. It was also observed that $\mathbf{5}$ exhibited 2.5-fold lower activity than $\mathbf{1}$ in MIA cells. Compounds $\mathbf{8}$ and 11 exhibited higher activity than $\mathbf{3}$ and $\mathbf{2}$ respectively across all cancer cell lines. In a similar vein, compound 14 was more active than 4 in all cells, except MCF-7, A2780 and H460 cells. The dimethyltin(IV) $(6,9,12$, and 15) and tin(IV) $(\mathbf{1 0}, \mathbf{1 3}$, and 16) compounds exhibited no significant difference as compared to their Schiff bases. It can be concluded that the presence of two phenyl groups attached to the tin atom at the center improved cytotoxicity against all tested cancer cell lines. The planarity of the aromatic $\pi$ system makes it available for stacking and easier penetration into the double helix of the DNA of cancer cells [78]. Overall, the cytotoxicity data indicated that HT29, MCF-7, A2780, A431, BE2-C, and MIA were more sensitive, whereas H460 and DU145 cells were more resistant against the Schiff bases and tin(IV) compounds than cisplatin that were investigated in this study. By comparing the toxicity of the compounds tested, all tin(IV) compounds (5-16) showed lower toxicity in MCF10A cells as the $\mathrm{GI}_{50}$ values of MCF10A were higher (less active) than the $\mathrm{GI}_{50}$ values of certain cancer cells. However, MCF10A is positive for telomerase reverse transcriptase [79] which is known to be up-regulated in many cancer cells as well. The decrease in cell viability after treatment with synthesised compounds may be due to the inactivation of this enzyme. The use of MCF10A in this study is to act as a benchmark for the cytotoxicity data obtained [80]. 
Table 1. In vitro cytotoxicity of tin(IV) compounds (5-16) derived from thiosemicarbazone Schiff bases (1-4) against several cell lines, determined by MTT assay and expressed as $\mathrm{GI}_{50}$ values with standard error. $\mathrm{GI}_{50}$ is the concentration at which cell growth is inhibited by $50 \% 72$ hours post-incubation.

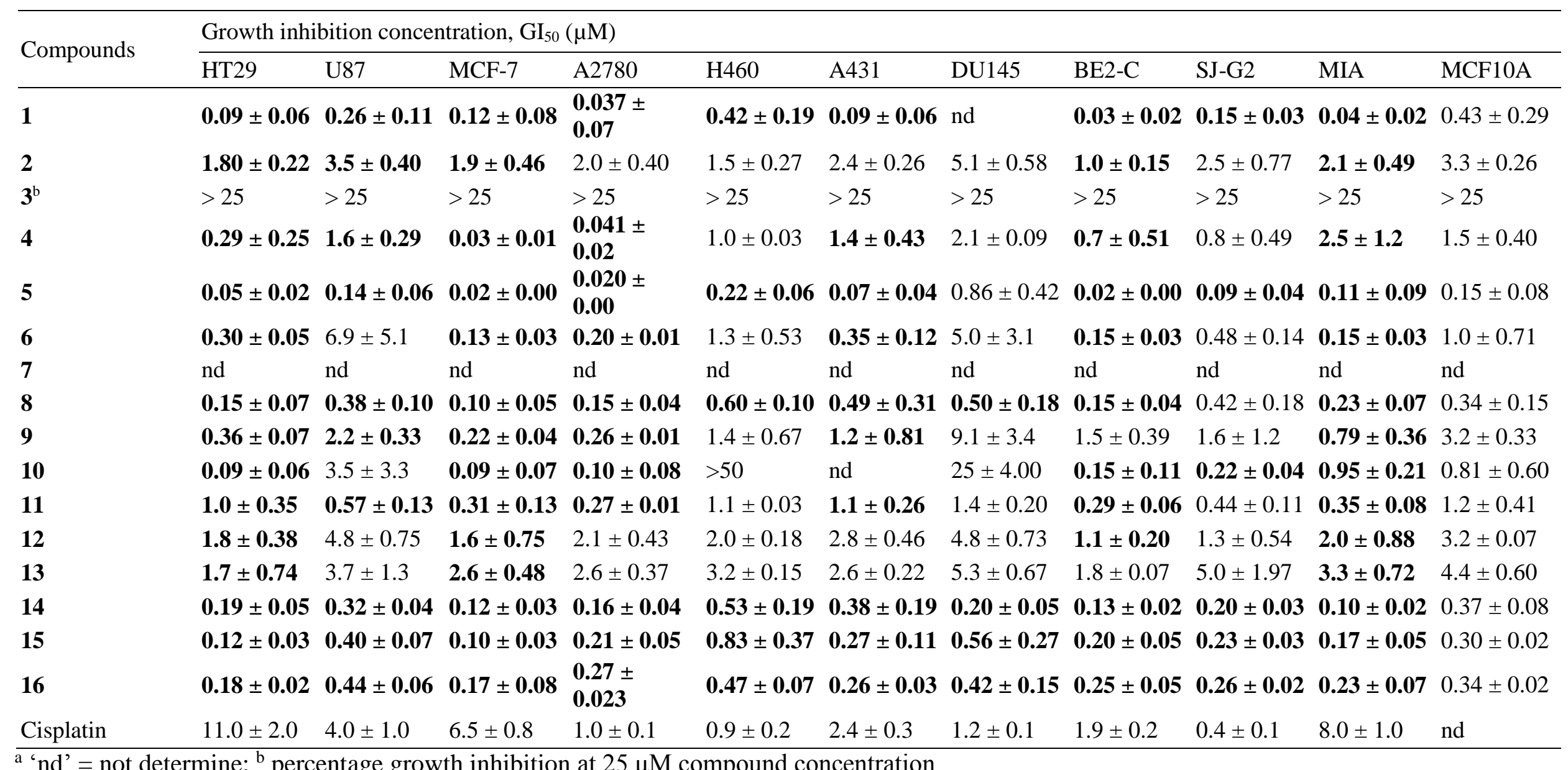




\section{Conclusions}

A series of twelve tin(IV) compounds derived from four thiosemicarbazone Schiff bases have been synthesized and characterized by physicochemical and spectroscopic techniques as well as X-ray crystallographic analysis. X-ray crystallography indicated a highly distorted coordination geometry trigonal-bipyramidal for $\mathrm{Ph}_{2} \mathrm{Sn}\left(\mathrm{L}^{1}\right)$ in $\mathbf{1 1}^{\prime}$ and for $\mathrm{Me}_{2} \mathrm{Sn}\left(\mathrm{L}^{1}\right)$ in 12. An interesting pattern of cytotoxicity was observed where compound $\mathbf{1}$ was selectively active against HT29, A2780, A431, BE2-C and MIA, while $\mathbf{3}$ was inactive against all cancer cells. Both were similar in structure, the difference being the methoxy (1) and hydroxyl (3) substituent at the meta position of the phenyl ring. In contrast, compound $\mathbf{4}$ having a hydroxyl group at phenyl ring demonstrated greater activity than compound $\mathbf{2}$ which had a methoxy group. Diphenyltin(IV) compound 5 displayed excellent activity in the range of $0.016-0.22$ $\mu \mathrm{M}$ against all the cancer cells tested. Overall, diphenyltin(IV) compounds showed the most promising anticancer potential. Based on findings in this study, thiosemicarbazone Schiff bases and their tin(IV) compounds have significant anticancer potential and further mechanism of action and in vivo studies are required to determine the action of these compounds in vivo for better intracellular understanding.

Funding: This research was funded by Universiti Putra Malaysia under the Geran Putra IPS (9504600) and Geran Putra IPB (9581001) (UPM) and the Malaysian Fundamental Research Grant Scheme (FRGS No. 01-01-16- 1833FR).

Acknowledgements: We thank the Department of Chemistry, the Molecular Genetics Laboratory and the Department of Biomedical Sciences, Universiti Putra Malaysia, Malaysia and the Discipline of Chemistry, University of Newcastle and the Calvary Mater Hospital, Australia for their facilities. Sunway University $\mathrm{Sdn}$ Bhd is thanked for X-ray crystallographic analysis and financial support (Grant. no. STR-RCTR-RCCM-001-2019). E.N.M.Y. wishes to thank Ministry of Higher Education Malaysia for the award of MyPhD, MyBrain15 and the University of Newcastle for the award of University of Newcastle International Postgraduate Research Scholarship. We would also like to thank Karen A. Crouse, Robert Burns and Adam McCluskey for helpful discussions. 
Conflicts of Interest: The authors declare no conflict of interest.

\section{References}

[1] G. Domagk, R. Behnisch, F. Mietzsch, H. Schimidt, Naturwissenschaften. 33 (1946) 315.

[2] H. Beraldo, D. Gambino, Mini Rev. Med. Chem. 4 (2004) 31-39.

[3] H.L. Singh, A.K. Varshney, Appl. Organomet. Chem. 15 (2001) 762-768.

[4] E.C. Moore, M.S. Zedeck, K.C. Agrawal, A.C. Sartorelli, Biochemistry. 9 (1970) 4492-4498.

[5] K. Fent, Crit. Rev. Toxicol. 26 (1996) 1-117.

[6] M. Jain, S. Gaur, V.P. Singh, R. V Singh, Main Gr. Met. Compd. 18 (2004) 73-82.

[7] K. Liu, H. Yan, G. Chang, Z. Li, M. Niu, M. Hong, Inorganica Chim. Acta. 464 (2017) 137-146.

[8] M.A. Salam, M.A. Hussein, I. Ramli, S. Islam, J. Organomet. Chem. 813 (2016) 7177.

[9] R. Singh, N.K. Kaushik, Spectrochim. Acta - Part A Mol. Biomol. Spectrosc. 71 (2008) 669-675.

[10] M. Tariq, S. Ali, N. Muhammad, N.A. Shah, M. Sirajuddin, M.N. Tahir, N. Khalid, M.R. Khan, J. Coord. Chem. 67 (2014) 323-340.

[11] A. Kumar, P. Chaudhary, R. Singh, N.K. Kaushik, Main Gr. Chem. 15 (2016) 163178.

[12] A. Bacchi, A. Bonardi, M. Carcelli, P. Mazza, P. Pelagatti, C. Pelizzi, G. Pelizzi, C. Solinas, F. Zani, J. Inorg. Biochem. 69 (1998) 101-112.

[13] Y.F. Win, C.S. Choong, J.C. Dang, M.A. Iqbal, C.K. Quah, A.M.S.A. Majid, S.G. Teoh, J. Coord. Chem. 67 (2014) 3401-3413.

[14] R. Malhotra, A. Ravesh, V. Singh, Phosphorus. Sulfur. Silicon Relat. Elem. 192 (2017) 73-80.

[15] M. Sirajuddin, S. Ali, V. Mckee, M. Sohail, H. Pasha, Eur. J. Med. Chem. 84 (2014) 343-363.

[16] T. Sedaghat, A. Golalzadeh, H. Motamedi, Phosphorus, Sulfur Silicon Relat. Elem. 188 (2013) 1694-1702.

[17] M. Gielen, Appl. Organomet. Chem. 16 (2002) 481-494.

[18] M. Gielen, M. Biesemans, R. Willem, Appl. Organomet. Chem. 19 (2005) 440-450.

[19] H.L. Singh, J.B. Singh, S. Bhanuka, Res. Chem. Intermed. 42 (2016) 997-1015. 
[20] M. Khandani, T. Sedaghat, N. Erfani, M.R. Haghshenas, H.R. Khavasi, J. Mol. Struct. 1037 (2013) 136-143.

[21] J. Wiecek, V. Dokorou, Z. Ciunik, D. Kovala-Demertzi, Polyhedron. 28 (2009) 32983304.

[22] M.A. Salam, M.A. Affan, M.A. Arafat, R. Saha, R. Nasrin, Heteroat. Chem. 24 (2013) 43-52.

[23] R.A. Haque, M.A. Salam, M.A. Arafath, J. Coord. Chem. 68 (2015) 2953-2967.

[24] M.A. Salam, A. Arafath, M.A. Hussein, R. Basri, R. Pervin, Phosphorus. Sulfur. Silicon Relat. Elem. 191 (2016) 1101-1107.

[25] E.N.M. Yusof, M.A.M. Latif, M.I.M. Tahir, J.A. Sakoff, M.I. Simone, A.J. Page, A. Veerakumarasivam, E.R.T. Tiekink, T.B.S.A. Ravoof, Int. J. Mol. Sci. 20 (2019) 854.

[26] E.N.M. Yusof, M.A.M. Latif, M.I.M. Tahir, J.A. Sakoff, A. Veerakumarasivam, A.J. Page, E.R.T. Tiekink, T.B.S.A. Ravoof, J. Mol. Struct. 1205 (2020) 127635.

[27] P. Kalaivani, R. Prabhakaran, E. Ramachandran, F. Dallemer, G. Paramaguru, R. Renganathan, P. Poornima, V. Vijaya Padma, K. Natarajan, Dalt. Trans. 41 (2012) $2486-2499$.

[28] I. Đilović, M. Rubčić, V. Vrdoljak, S.K. Pavelić, M. Kralj, I. Piantanida, M. Cindrić, Bioorg. Med. Chem. 16 (2008) 5189-5198.

[29] A.T. Swesi, Y. Farina, I. Baba, Sains Malaysiana. 36 (2007) 21-26.

[30] A.T. Swesi, Y. Farina, M. Kassim, S.W. Ng, Acta Crystallogr. Sect. E Struct. Reports Online. E62 (2006) o5457-05458.

[31] Rigaku Oxford Diffraction. CrysAlis PRO. Agilent Technologies Inc., Santa Clara, CA, USA 2015.

[32] G.M. Sheldrick, Acta Crystallogr. Sect. C Struct. Chem. C71 (2015) 3-8.

[33] G.M. Sheldrick, Acta Crystallogr. Sect. A Found. Crystallogr. A64 (2008) 112-122.

[34] L.J. Farrugia, J. Appl. Crystallogr. 45 (2012) 849-854.

[35] K. Brandenburg, DIAMOND, Crystal Impact GbR, (2006).

[36] A.L. Spek, Acta Crystallogr. Sect. E Crystallogr. Commun. E76 (2020) 1-11.

[37] M.J. Frisch, G.W. Trucks, H.B. Schlegel, G.E. Scuseria, M.A. Robb, J.C. R., G. Scalmani, V. Barone, G.A. Petersson, H. Nakatsuji, X. Li, M. Caricato, A. Marenich, J. Bloino, B.G. Janesko, R. Gomperts, B. Mennucci, H.P. Hratchian, J. V. Ortiz, A.F. Izmaylov, J.L. Sonnenberg, D. Williams-Young, F. Ding, F. Lipparini, F. Egidi, J. Goings, B. Peng, A. Petrone, T. Henderson, D. Ranasinghe, V.G. Zakrzewski, J. Gao, N. Rega, G. Zheng, W. Liang, M. Hada, M. Ehara, K. Toyota, R. Fukuda, J. Hasegawa, M. Ishida, T. Nakajima, Y. Honda, O. Kitao, H. Nakai, T. Vreven, K. Throssell, J.A. Montgomery, Jr., J.E. Peralta, F. Ogliaro, M. Bearpark, J.J. Heyd, E. Brothers, K.N. Kudin, V.N. Staroverov, T. Keith, R. Kobayashi, J. Normand, K. Raghavachari, A. Rendell, J.C. Burant, S.S. Iyengar, J. Tomasi, M. Cossi, J.M. 
Millam, M. Klene, C. Adamo, R. Cammi, J.W. Ochterski, R.L. Martin, K. Morokuma, O. Farkas, J.B. Foresman, D.J. Fox, Gaussian 09, Revision D.01, Wallingford CT. (2013).

[38] D. Roy, K. Todd, M. John, GaussView, Ver 5.0.9, (2009).

[39] C. Lee, W. Yang, R.G. Parr, Phys. Rev. B. 37 (1988) 785-789.

[40] A.D. Becke, J. Chem. Phys. 98 (1993) 5648-5652.

[41] P.J. Hay, W.R. Wadt, J. Chem. Phys. 82 (1985) 270-283.

[42] P.J. Hay, W.R. Wadt, J. Chem. Phys. 82 (1985) 299-310.

[43] W.R. Wadt, P.J. Hay, J. Chem. Phys. 82 (1985) 284-298.

[44] P.M. Jeffrey, M. Damian, L. Radom, J. Phys. Chem. A. 111 (2007) 11683-11700.

[45] K.-Y. Chen, H.-Y. Tsai, Int. J. Mol. Sci. 15 (2014) 18706-18724.

[46] G. Scalmani, M.J. Frisch, B. Mennucci, J. Tomasi, R. Cammi, V. Barone, J. Chem. Phys. 124 (2006) 94107.

[47] E. Cancès, B. Mennucci, J. Tomasi, J. Chem. Phys. 107 (1997) 3032-3041.

[48] J. Tomasi, M. Persico, Chem. Rev. 94 (1994) 2027-2094.

[49] J. Tomasi, B. Mennucci, R. Cammi, Chem. Rev. 105 (2005) 2999-3093.

[50] L.R. Odell, M.K. Abdel-Hamid, T.A. Hill, N. Chau, K.A. Young, F.M. Deane, J.A. Sakoff, S. Andersson, J.A. Daniel, P.J. Robinson, A. McCluskey, J. Med. Chem. 60 (2017) 349-361.

[51] J. Gilbert, G.N. De Iuliis, M. Tarleton, A. McCluskey, J.A. Sakoff, Mol. Pharmacol. 93 (2018) 168-177.

[52] J.A. Sakoff, S.P. Ackland, Cancer Chemother. Pharmacol. 46 (2000) 477-487.

[53] A.M. Bergman, V.W.T.R. van Haperen, G. Veerman, C.M. Kuiper, G.J. Peters, Clin. Cancer Res. 2 (1996) 521-530.

[54] V. Vrdoljak, M. Cindrić, D. Milić, D. Matković-Čalogović, P. Novak, B. Kamenar, Polyhedron. 24 (2005) 1717-1726.

[55] F.V. Rocha, C.V. Barra, A.E. Mauro, I.Z. Carlos, L. Nauton, M. El Ghozzi, A. Gautier, L. Morel, A.V.G. Netto, Eur. J. Inorg. Chem. (2013) 4499-4505.

[56] W.J. Geary, Coord. Chem. Rev. 7 (1971) 81-122.

[57] P.M. Krishna, B.S. Shankara, N.S. Reddy, Hindawi. (2013) 1-11.

[58] Y. Matsuda, T. Ebata, N. Mikami, J. Chem. Phys. 110 (1999) 8397.

[59] F. Wang, H. Yin, J. Cui, Y. Zhang, H. Geng, M. Hong, J. Organomet. Chem. 759 (2014) 83-91. 
[60] J.M. Galván-Hidalgo, G.M. Chans, T. Ramírez-Apan, A. Nieto-Camacho, S. Hernández-Ortega, E. Gómez, Appl. Organomet. Chem. (2017) 1-12.

[61] Naqeebullah, Y. Farina, K.M. Chan, L.K. Mun, N.F. Rajab, T.C. Ooi, Molecules. 18 (2013) 8696-8711.

[62] H.B. Shawish, M. Paydar, C.Y. Looi, Y.L. Wong, E. Movahed, S.N.A. Halim, W.F. Wong, M.R. Mustafa, M.J. Maah, Transit. Met. Chem. 39 (2014) 81-94.

[63] J. Holeček, M. Nádvorník, K. Handlír, A. Lyčka, J. Organomet. Chem. 315 (1986) 299-308.

[64] X. Shang, X. Meng, E.C.B.A. Alegria, Q. Li, C. Guedes, M.L. Kuznetsov, A.J.L. Pombeiro, Inorg. Chem. 50 (2011) 8158-8167.

[65] M. Nath, P.K. Saini, A. Kumar, J. Organomet. Chem. 695 (2010) 1353-1362.

[66] M. Hong, G. Chang, R. Li, M. Niu, New J. Chem. 40 (2016) 7889-7900.

[67] S. Yadav, I. Yousuf, M. Usman, M. Ahmad, F. Arjmand, S. Tabassum, RSC Adv. 5 (2015) 50673-50690.

[68] M. Sirajuddin, S. Ali, M.N. Tahir, Inorganica Chim. Acta. 439 (2016) 145-158.

[69] A.W. Addison, T.N. Rao, J. Chem. Soc. Dalt. Trans. 7 (1984) 1349-1356.

[70] M. Rubčić, I. Dstrokeilović, M. Cindrić, D. Matković-Čalogović, Acta Crystallogr. Sect. C Cryst. Struct. Commun. 64 (2008) o570-o573.

[71] L.R. Gomes, J.N. Low, N.R.D.L. Correira, T.C.M. Noguiera, A.C. Pinheiro, M.V.N. De Souza, J.L. Wardell, S.M.S.V. Wardell, Zeitschrift Fur Krist. - Cryst. Mater. 234 (2019) 59-71.

[72] R. Lu, W. Wang, X. Lü, S. Zhao, Acta Crystallogr. Sect. E Struct. Reports Online. 67 (2011) 02702 .

[73] E.R.T. Tiekink, Coord. Chem. Rev. 345 (2017) 209-228.

[74] D.P. Malenov, G. V. Janjić, V.B. Medaković, M.B. Hall, S.D. Zarić, Coord. Chem. Rev. 345 (2017) 318-341.

[75] N.W. Alcock, Adv. Inorg. Chem. Radiochem. 15 (1972) 1-58.

[76] E.R.T. Tiekink, Appl. Organomet. Chem. 5 (1991) 1-23.

[77] T.S. Basu Baul, A. Paul, L. Pellerito, M. Scopelliti, P. Singh, P. Verma, A. Duthie, D. de Vos, E.R.T. Tiekink, Invest. New Drugs. 29 (2011) 285-299.

[78] F. Arjmand, G.C. Sharma, F. Sayeed, M. Muddassir, S. Tabassum, J. Photochem. Photobiol. B Biol. 105 (2011) 167-174.

[79] B.J. Sishc, C.B. Nelson, M.J. Mckenna, C.L.R. Battaglia, C. Tanzarella, Front. Oncol. 5 (2015) 1-19.

[80] J.H. De Jong, H.M. Rodermond, C.D. Zimberlin, V. Lascano, F.D.S.E. Melo, D.J. Richel, J.P. Medema, L. Vermeulen, Sci. Rep. 2 (2012) 271. 
(a)
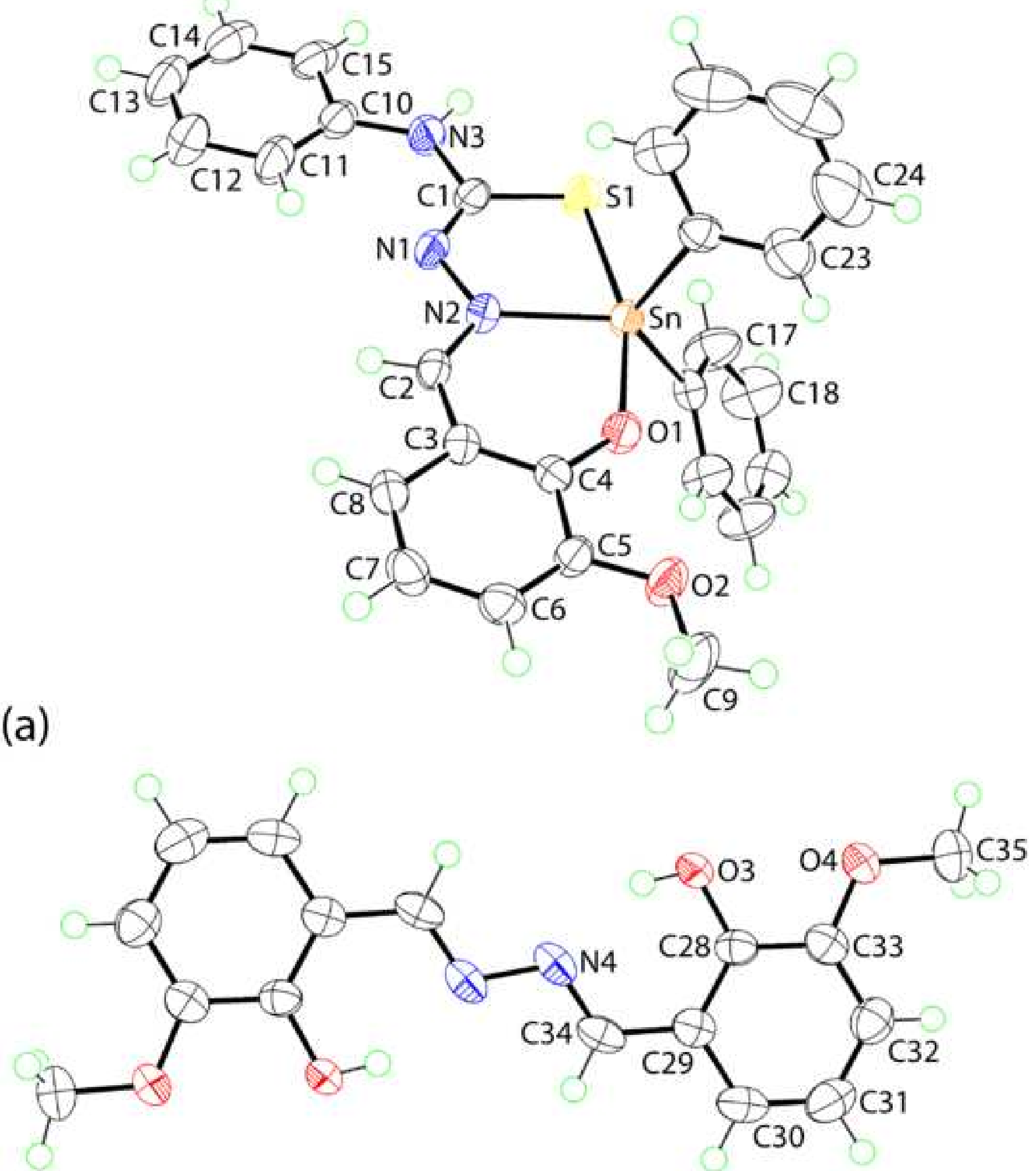

(b) 


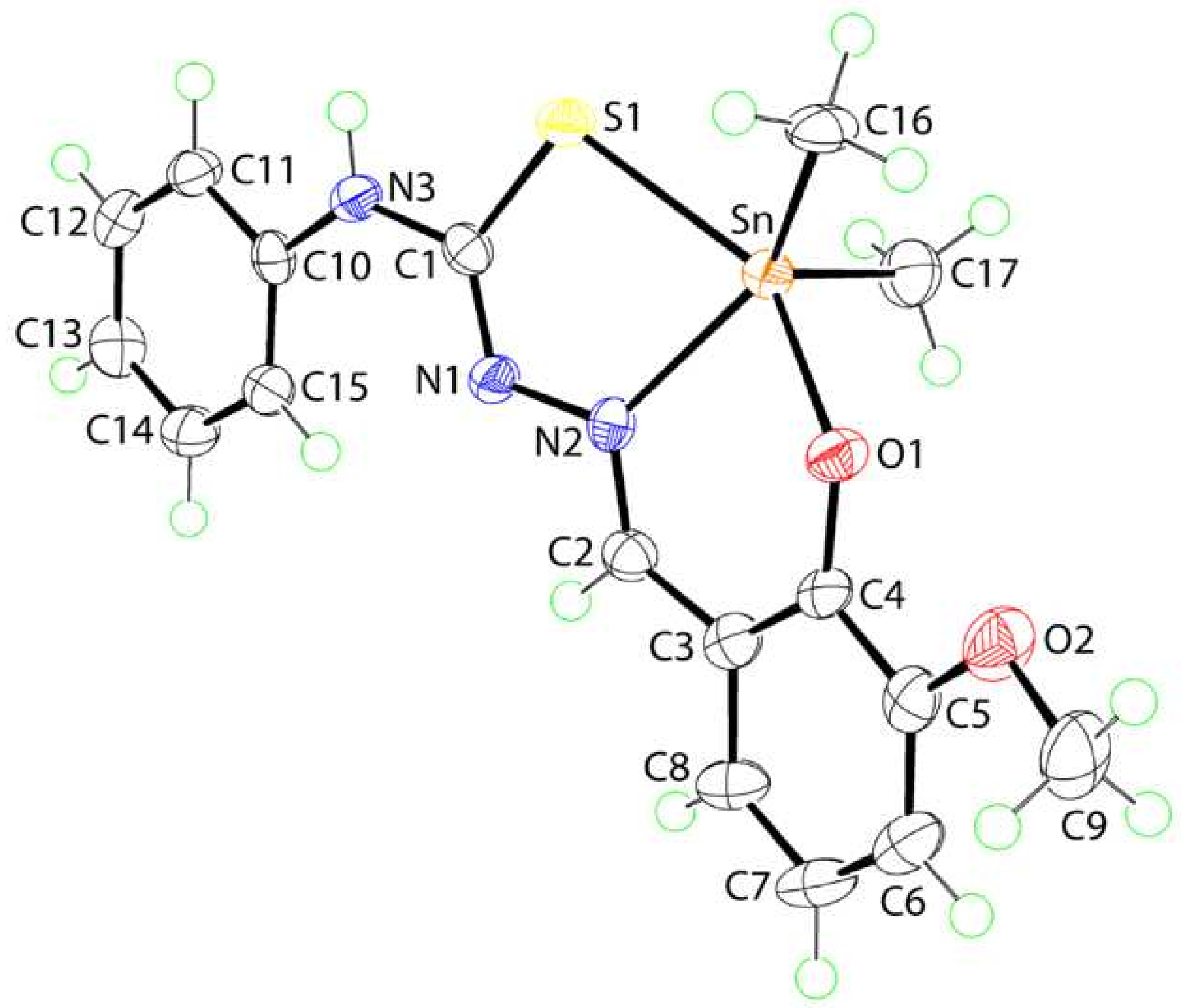



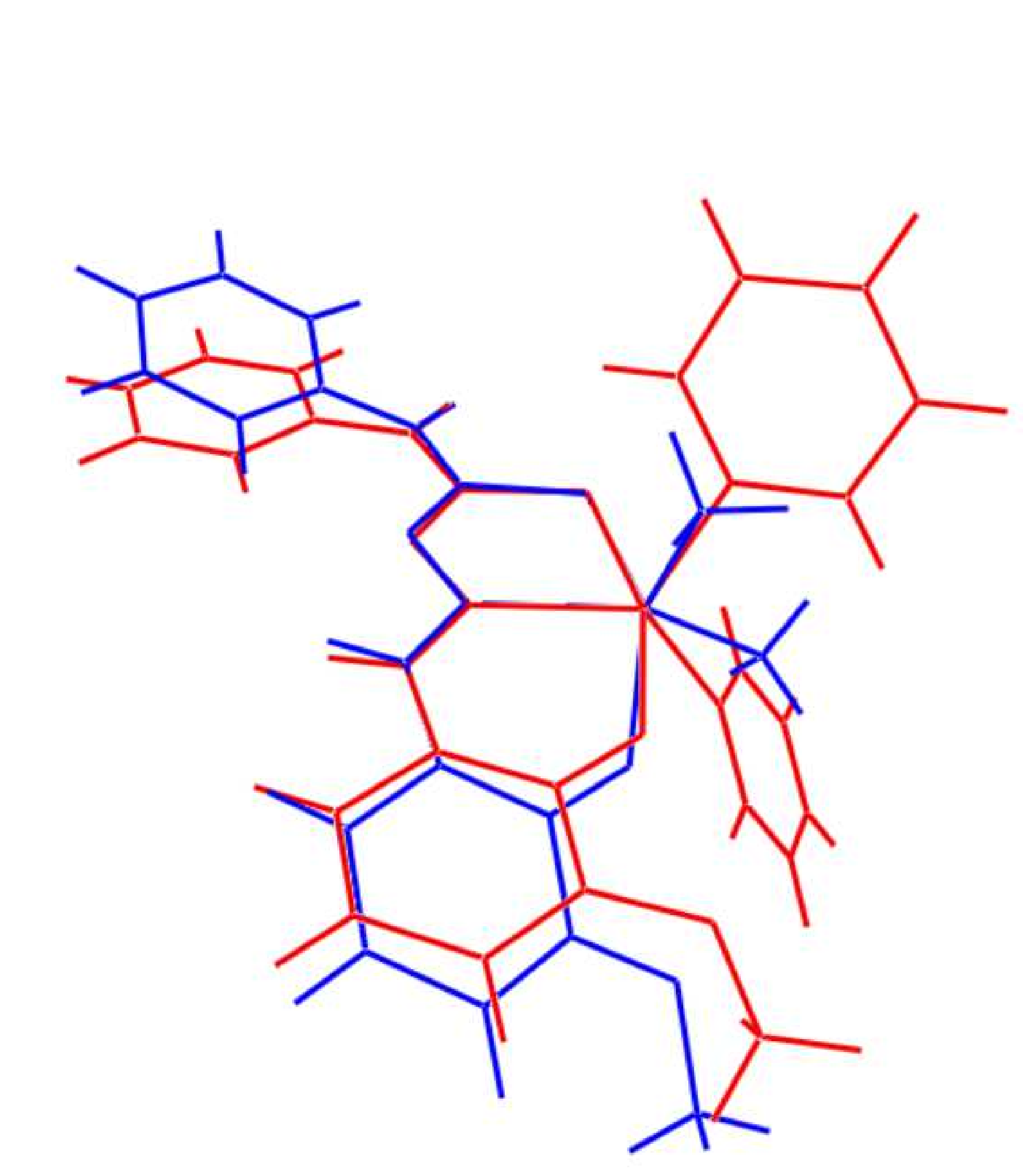

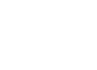

Figure 4

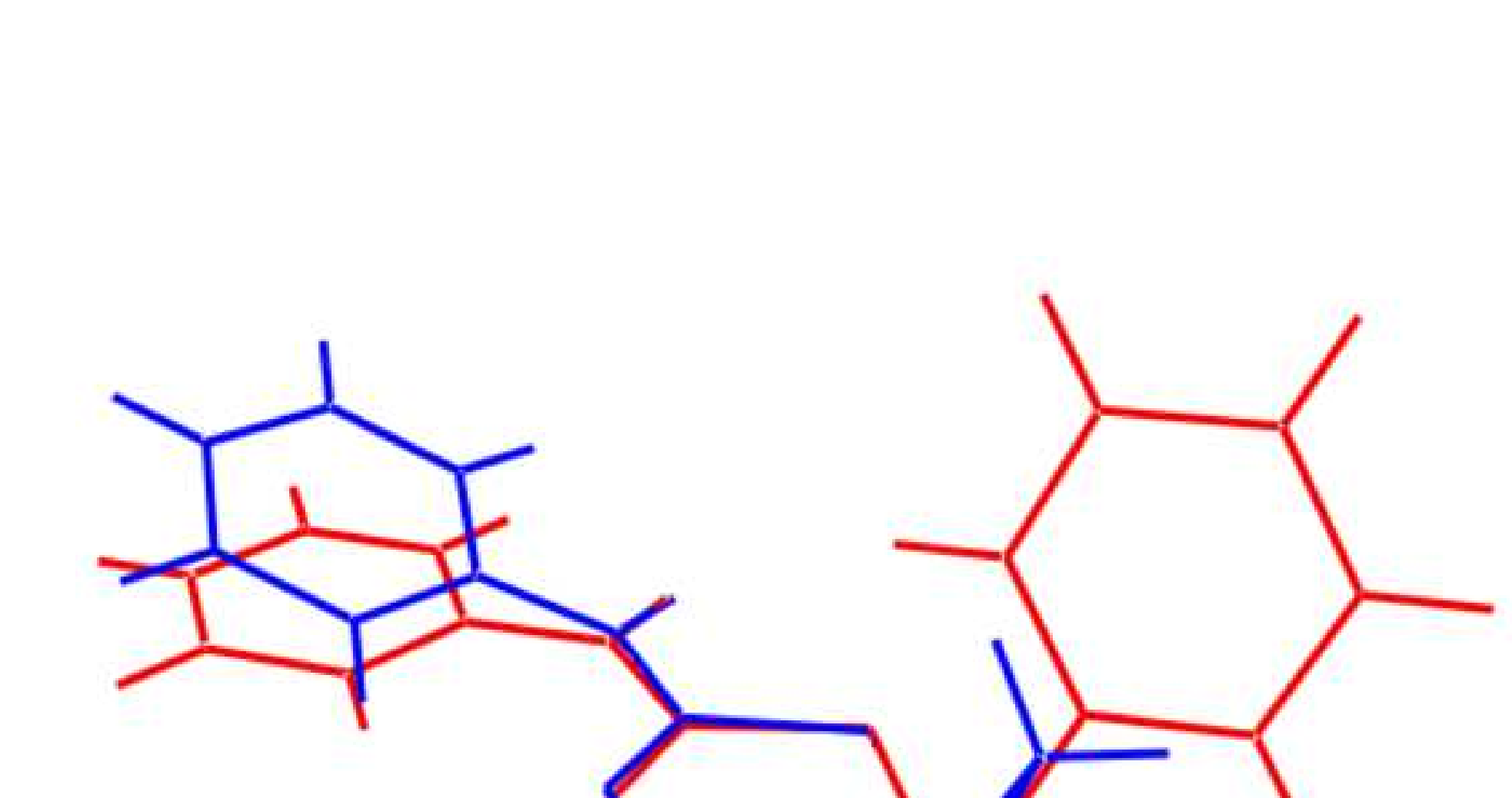

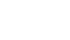

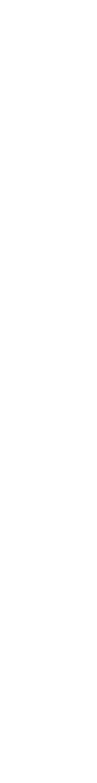


(a)

(c)
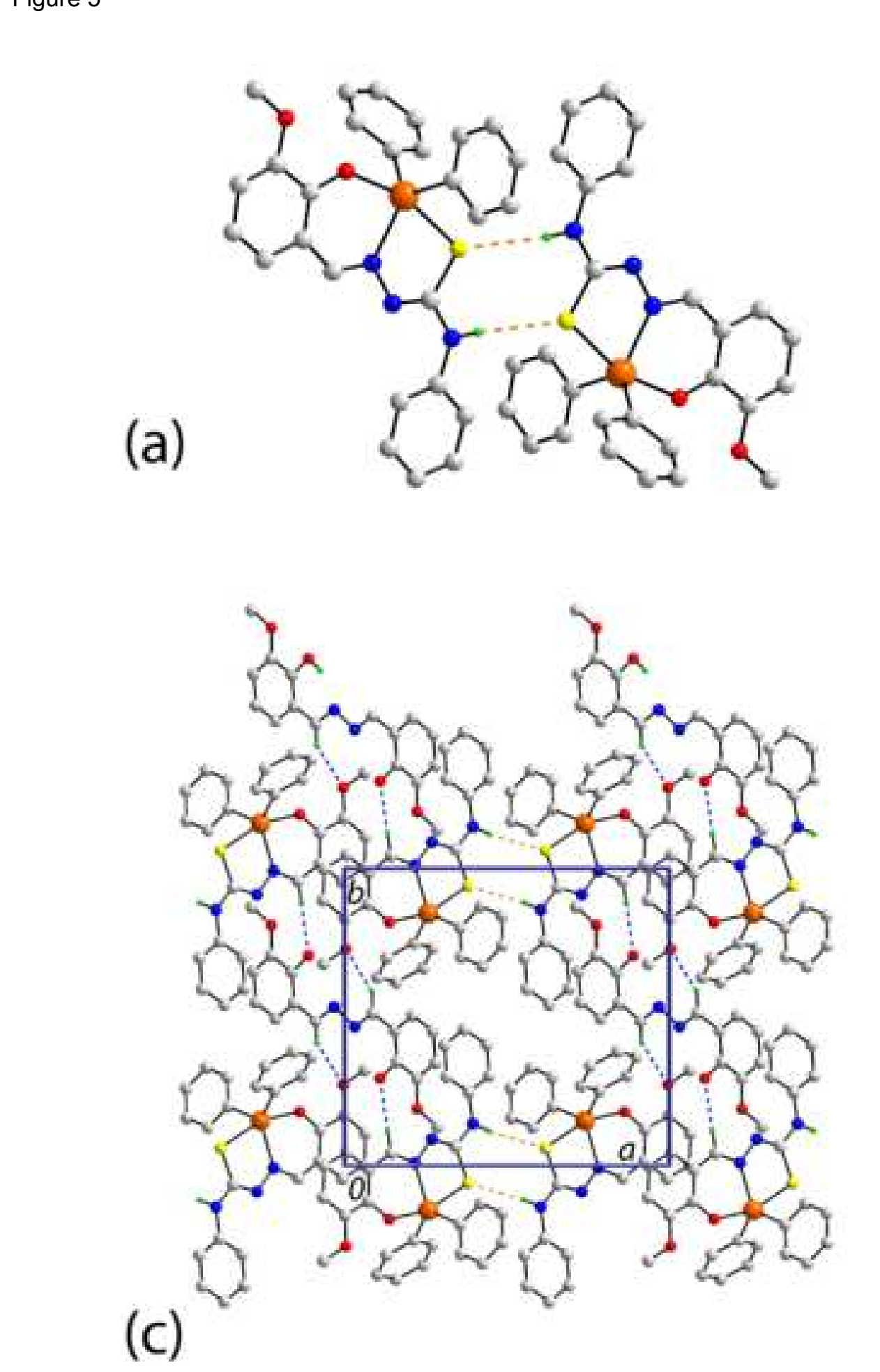

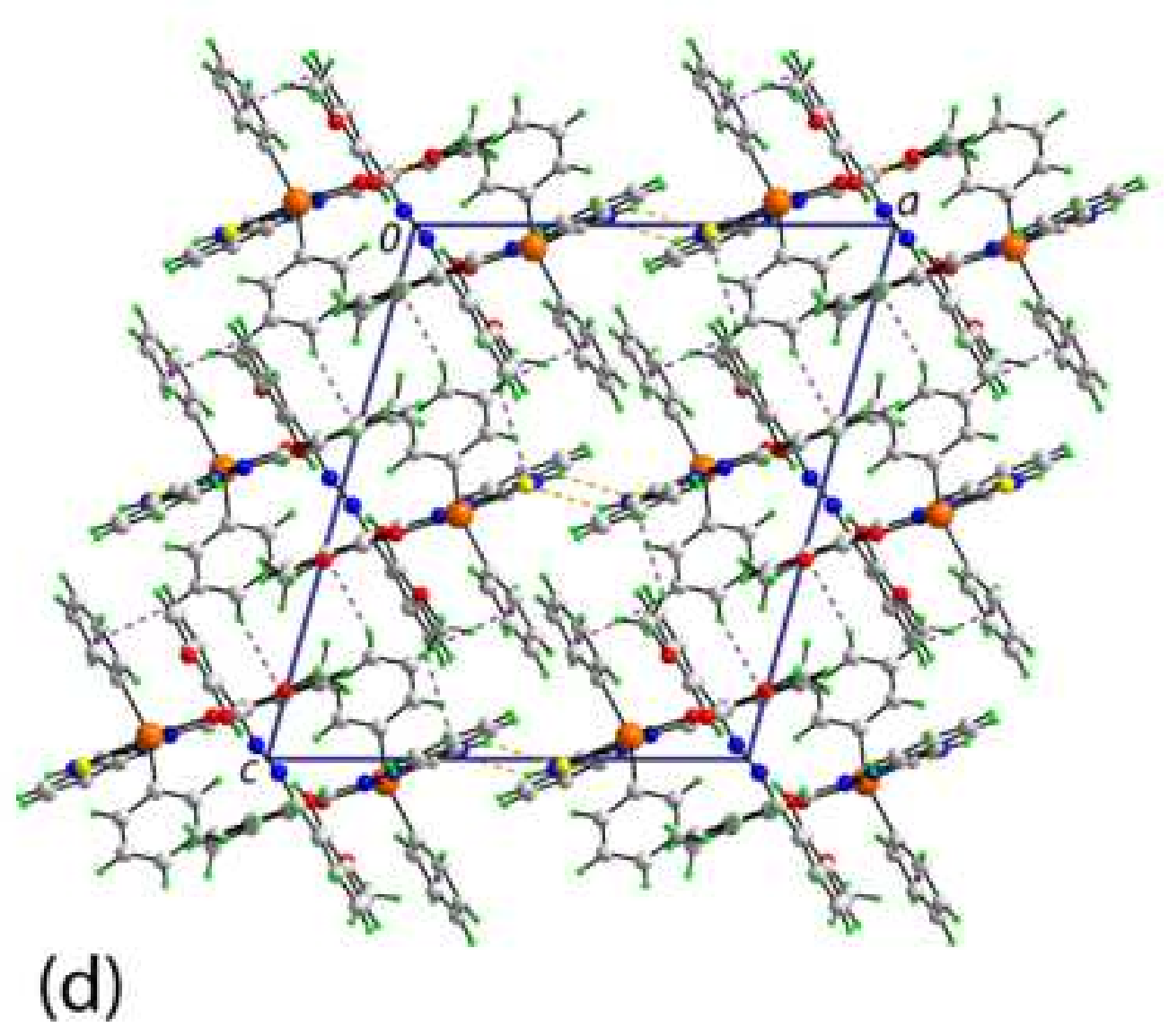

(d)

(b)

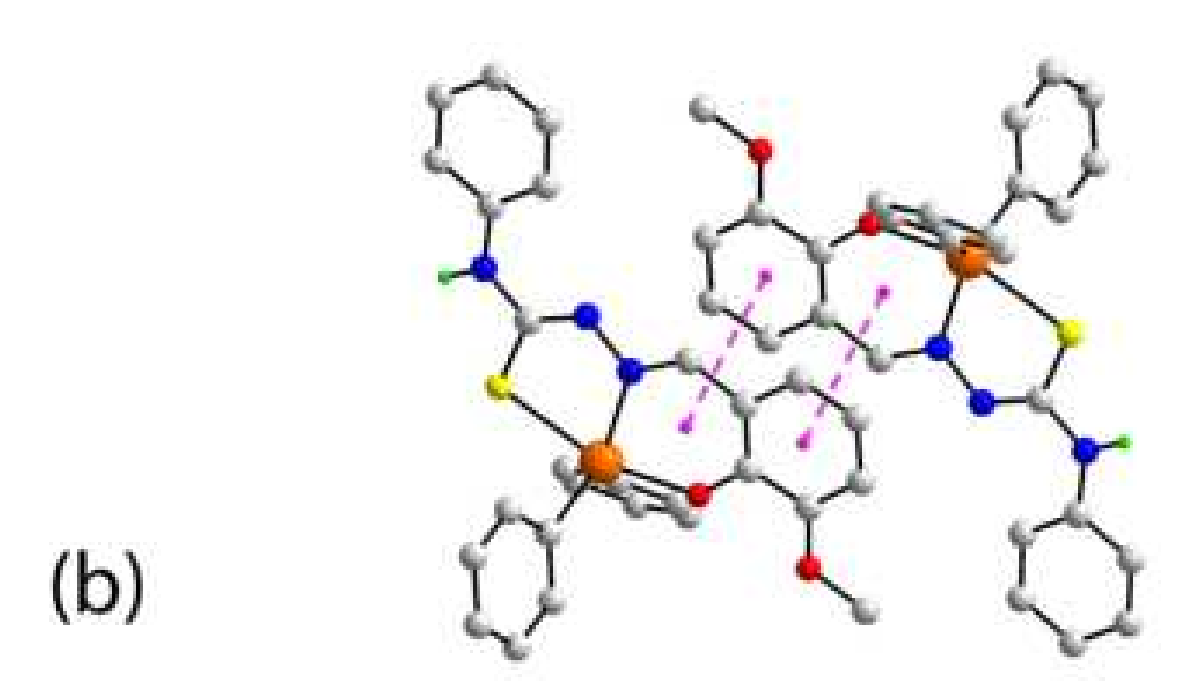


(a)
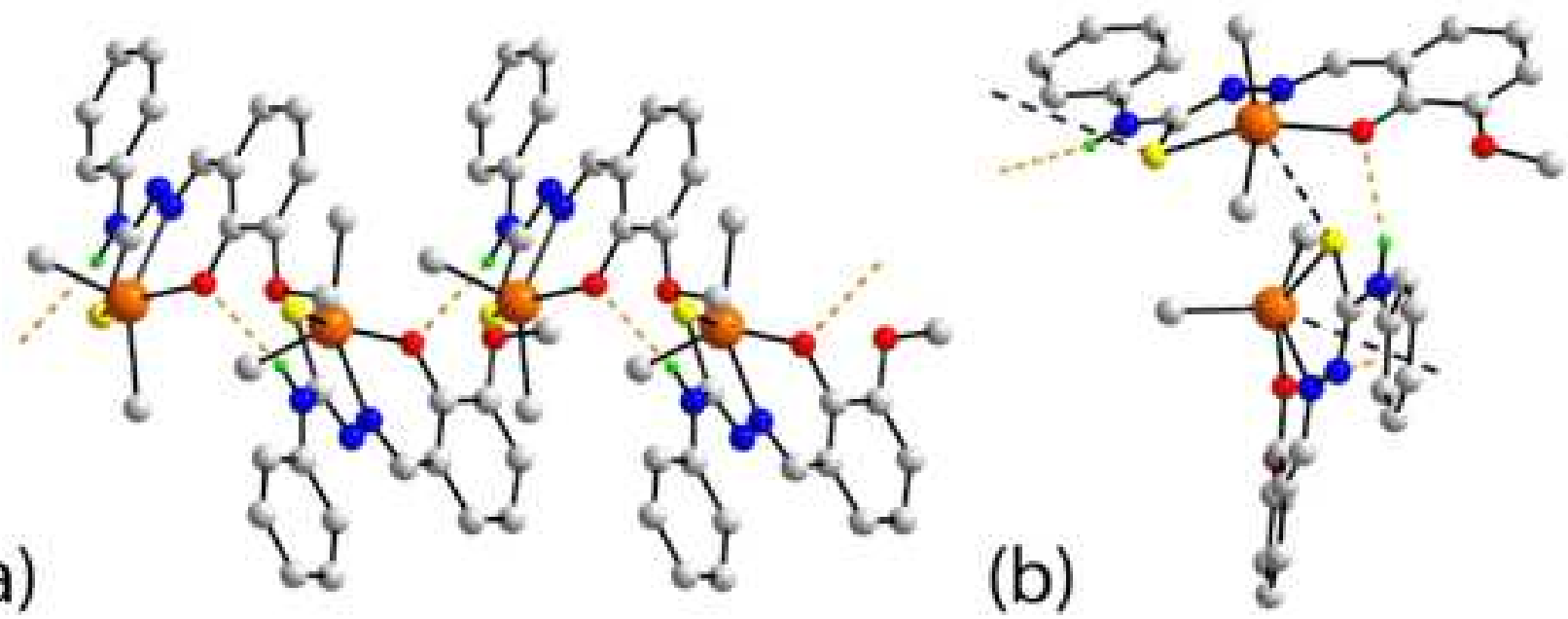

(b)
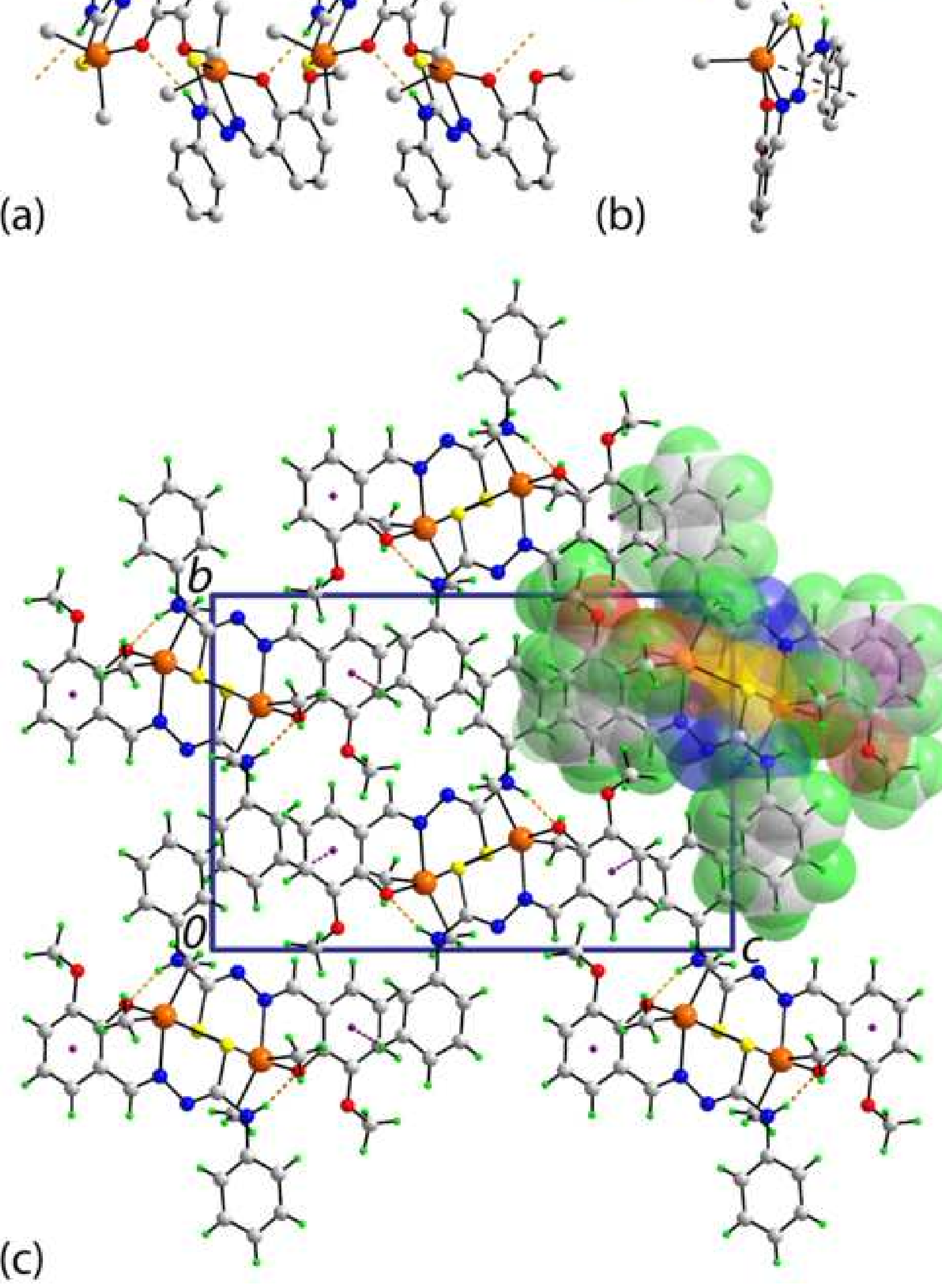


\section{Click here to access/download \\ CIF (*if crystal structure is described) \\ 11.cif}


CIF ( ${ }^{*}$ if crystal structure is described)

\section{Click here to access/download \\ CIF (*if crystal structure is described) \\ 12.cif}


CheckCIF ( ${ }^{*}$ if crystal structure is described)

\section{Click here to access/download \\ CheckCIF (*if crystal structure is described) 11_checkcif.pdf}


CheckCIF ( ${ }^{*}$ if crystal structure is described)

\section{Click here to access/download \\ CheckCIF (*if crystal structure is described) 12_checkcif.pdf}




\section{Click here to access/download \\ Supplementary Info for Reviewers Polyhedron_SI_v1.docx}




\section{Click here to access/download
DC Depository Request.pdf \\ Click here to access/download
Supplementary Info for Reviewers
CCDC Depository Request.pdf Click here to access/download
Supplementary Info for Reviewers
CCDC Depository Request.pdf} ra 


\section{Highlights}

- Thiosemicarbazone Schiff bases $\mathbf{1}$ and $\mathbf{4}$ are useful lead candidates for the future organic drug design development to treat cancers.

- Trigonal bipyramidal diphenyltin(IV) compounds 5, 8, 11 and $\mathbf{1 4}$ exhibited excellent cytotoxic activity against a panel of ten cancer cell lines tested but minimal toxicity against MCF-10A (normal breast). 


\section{Declaration of interests}

$\bigotimes$ The authors declare that they have no known competing financial interests or personal relationships that could have appeared to influence the work reported in this paper.

$\bigotimes$ The authors declare the following financial interests/personal relationships which may be considered as potential competing interests:

Nil 


\section{Credit Author Statement}

Enis Nadia Md Yusof Formal analysis, Investigation, Data Curation, Writing - Original Draft, Visualization

Alister J. Page Software, Formal analysis, Resources, Data Curation, Writing - Review \& Editing,, Visualization, Supervision, Project administration

Jennette A. Sakoff Formal analysis, Resources, Data Curation, Writing - Review \& Editing

Michela I. Simone Validation, Formal analysis, Data Curation, Writing - Review \& Editing

Abhi Veerakumarasivam Formal analysis, Resources, Data Curation, Writing - Review \& Editing, Supervision, Funding acquisition

Edward R. T. Tiekink Software, Formal analysis, Data Curation, Writing - Review \& Editing, Visualization

Thahira B. S. A. Ravoof Conceptualization, Methodology, Resources, Writing - Review \& Editing, Supervision, Project administration, Funding acquisition 